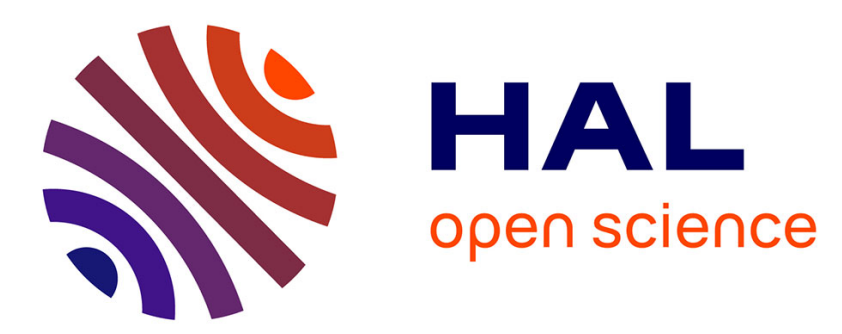

\title{
Optimal input experiment design and parameter estimation in core-scale pressure oscillation experiments
} M.G. Potters, M. Mansoori, Xavier Bombois, J.D. Jansen, P.M.J. van den Hof

\section{To cite this version:}

M.G. Potters, M. Mansoori, Xavier Bombois, J.D. Jansen, P.M.J. van den Hof. Optimal input experiment design and parameter estimation in core-scale pressure oscillation experiments. Journal of Environmental Hydrology, 2016, 534, pp.534-552. 10.1016/j.jhydrol.2016.01.043 . hal-01355013

\section{HAL Id: hal-01355013 \\ https://hal.science/hal-01355013}

Submitted on 25 Apr 2019

HAL is a multi-disciplinary open access archive for the deposit and dissemination of scientific research documents, whether they are published or not. The documents may come from teaching and research institutions in France or abroad, or from public or private research centers.
L'archive ouverte pluridisciplinaire HAL, est destinée au dépôt et à la diffusion de documents scientifiques de niveau recherche, publiés ou non, émanant des établissements d'enseignement et de recherche français ou étrangers, des laboratoires publics ou privés. 


\title{
Optimal Input Experiment Design and Parameter Estimation in Core-Scale Pressure Oscillation Experiments
}

\author{
M.G. Potters ${ }^{\mathrm{a}, *}$, M. Mansoori ${ }^{\mathrm{b}}$, X. Bombois ${ }^{\mathrm{c}}$, J.D. Jansen ${ }^{\mathrm{d}}$, P.M.J. Van den \\ Hof ${ }^{\mathrm{e}}$ \\ ${ }^{a}$ Delft Center for Systems and Control, Delft University of Technology, Mekelweg 2, 2628 \\ CD Delft, The Netherlands \\ ${ }^{b}$ Department of Chemical and Petroleum Engineering, Sharif University of Technology, \\ Azadi Ave, Tehran, Iran \\ ${ }^{c}$ Laboratoire Ampère UMR CNRS 5005, Ecole Centrale de Lyon, 36 Avenue Guy de \\ Collongue, 69134 Ecully Cedex, France \\ ${ }^{d}$ Faculty of Civil Engineering and Geosciences, Delft University of Technology, Stevinweg 1, \\ 2600 GA Delft, The Netherlands \\ ${ }^{e}$ Department of Electrical Engineering, Eindhoven University of Technology, P.O. Box 513, \\ $5600 \mathrm{MB}$, Eindhoven, The Netherlands
}

\begin{abstract}
This paper considers Pressure Oscillation (PO) experiments for which we find the minimum experiment time that guarantees user-imposed parameter variance upper bounds and honours actuator limits. The parameters permeability and porosity are estimated with a classical least-squares estimation method for which an expression of the covariance matrix of the estimates is calculated. This expression is used to tackle the optimization problem. We study the Dynamic Darcy Cell experiment set-up [1] and focus on data generation using square wave actuator signals, which, as we shall prove, deliver shorter experiment times than sinusoidal ones. Parameter identification is achieved using either inlet pressure/outlet pressure measurements [1] or actuator position/outlet pressure measurements, where the latter is a novel approach. The solution to the optimization problem reveals that for both measurement methods an optimal excitation frequency, an optimal inlet volume, and an optimal outlet volume exist. We find that under the same parameter variance bounds and actuator
\end{abstract}

*Corresponding author, m.g.potters@tudelft.nl

Preprint submitted to Journal of Hydrology

January 13, 2016 
constraints, actuator position/outlet pressure measurements result in required experiment times that are a factor fourteen smaller compared to inlet pressure/outlet pressure measurements. This result is analysed in detail and we find that the dominant effect driving this difference originates from an identifiability problem when using inlet-outlet pressure measurements for joint estimation of permeability and porosity. We illustrate our results with numerical simulations, and show excellent agreement with theoretical expectations.

Keywords: Experiment Design, Variance Constraints, Estimation, Porous

Media

\section{Introduction}

4

Two key parameters influencing fluid flow in a porous medium are permeability (i.e. inverse resistance) and porosity (i.e. storage capacity). These parameters are important to characterise fluid flow in underground water resources [2], contaminated water disposal in underground storages [3], and subsurface hydrocarbon reservoirs [4. Indeed, permeability and porosity estimates are used to initialise reservoir simulations, optimise the number of wells and their locations, and drilling and completion procedures.

At the core scale, estimation of both parameters locally may be carried out by performing an experiment on a cylindrically-shaped core sample of the porous medium, using either steady-state (SS), unsteady-state (USS), or pressure oscillation (PO) measurements. In an SS experiment a constant pressure difference is applied across the axis of the core sample and subsequently the flow rate is measured after the SS condition has been established. Permeability is then estimated based on the relationship between the flow rate and the pressure drop. In an USS experiment an impulse or step pressure change is applied at the upstream side of the sample while the pressure change is recorded downstream. The observed response is then analysed either graphically or numerically to estimate either permeability or porosity. Similarly, in a PO experiment, the recorded downstream pressure response is analyzed for parameter estimation - 
the difference being that an oscillatory pressure signal is applied upstream. The attenuation and phase shift between the up- and downstream signals are then translated into parameter estimates [5, 1. The oscillatory signal is usually a single sinusoid with a frequency and amplitude specified by the experimenter. The amplitude of the upstream signal is however bounded by the limits of the actuator. In cases where a rather high actuator frequency is necessary to take into account geometrical and physical properties of the sample, 6] suggested the use of input signals with complex shapes including the required high frequencies.

The consensus in the literature is that a PO experiment has several advantageous properties not shared by its SS and USS counterparts, e.g., less experiment time, less stress on the core sample, and the possibility of simultaneously estimating permeability and porosity [7, 3]. The effectiveness of PO experiments for the estimation of permeability has been demonstrated in different set-ups [1, 4, 8, 9, 6. Despite its advantageous properties, however, it is observed that measurements can result in large uncertainties in the estimates, particularly for porosity [7, 3, 4. Porosity estimates with an uncertainty exceeding an order of magnitude, or that have negative values, have been reported [3, 7]. (Negative values can however be easily circumvented by using log-transformed parameters). One cause is measurement noise, but in this paper we show that other ones also play an important role.

Furthermore, it is important to be able to reduce the experiment time without loss of accuracy. In such a case, more core samples can be analysed in a given time, which consequently reduces the experiment costs. Analogously, given a maximum experiment time, it is important to get the best possible estimates.

Clearly, the challenge of estimating permeability and porosity with high accuracy remains, especially in evaluating the production potential of tight formations in unconventional hydrocarbon reservoirs [4] or the sealing characteristics of the cap rock in underground storage [3].

Motivated by the above problems we raise the question whether we can, for a PO experiment, design the applied upstream pressure signal and utilise the 
degrees of freedom (DOF) in the experiment set-up in order to increase parameter accuracies. The dependence of the accuracy of the estimates on the selected driving frequency has been first pointed out in [10], although no investigation into this topic was pursued. From this question, we define the following optimization problem: find the minimal experiment time required to guarantee user-imposed variance constraints on the estimates by utilising DOF in the experiment set-up as well as designing the to-be-applied upstream pressure signal, ensuring that this signal has an amplitude that honours the actuator limits. Note that the solution can also be used to maximise the accuracy of the estimates for a given experiment length. To address this optimization problem we use techniques from Experiment Design.

Experiment Design addresses the long-standing issue of the lack of accurate parameter estimates inferred from collected data, particularly at the catchment scale. This issue is widely recognised; see for instance [11, 12, 13, 14, 15] and the nice review of [16. Some of the earliest works [17, 18, 19] in Experiment Design (although not recognised under this name at that time) showed that concepts such as parameter correlation, identifiability, observability, and experiment length strongly affect the quality of the parameter estimates (i.e. their variances). These works and those of [20, 21] were some of the first to quantitatively evaluate parameter uncertainty within a Bayesian framework. They provided measures to find the best possible calibration data for computer models, using a-posteriori data, i.e. data from an experiment that had already taken place. Other works [14, 22, analysed the role of tracer observations that influence parameter identifiability, and identifiability of unknown pollution sources. The works $23,24,25$, were the first to consider optimal experiment design for groundwater hydrology prior to the actual inference experiment; they mainly searched for optimal pumping and observation wells, keeping the pumping rates constant, such that the experiment cost could be minimized subject to maximizing the overall accuracy in the parameters (using a D-optimality criterion). More recently, a Bayesian methodology [26] was developed to find the optimal 
investigation strategy, or sampling pattern, prior to the actual experimental campaign.

We will take a non-Bayesian approach from linear systems theory [27] and apply it to the core-scale PO experiment introduced above. The method is different to the Bayesian methods in the sense that an optimal spectrum of the input signal is calculated prior to the actual experiment, whereas in the above methods the spectra of the inputs are not design variables. This optimal spectrum reveals e.g. the time scales that are important for accurate parameter estimation. We also consider variance constraints on the individual parameters, which is particularly important to use for systems that have low sensitivities for some parameters (in which case the D-optimality criterion, as used by e.g. [24], can be ill-chosen).

In this paper, we tackle the experiment design problem as follows. We perform parameter estimation using ordinary least squares using the measured noise-corrupted downstream pressure signal [28, 29]. This signal is deduced from the governing equations and boundary conditions, and depends on the applied upstream signal. One benefit of this method is that it can deal in a rather easy manner with (coloured) measurement noise; see [28] for details. A second benefit is that a frequency-domain expression of the covariance matrix of the estimates can be formulated. This expression, which we introduce in Section 3 is a function of the power spectrum of the applied signal and the DOF of the experiment set-up. Consequently, we can formulate the above optimization problem (of minimising the experiment time subject to parameter variance constraints and actuator bounds by designing the optimal input signal and DOF of the set-up) mathematically. We shall limit ourselves to sinusoidal and square-wave actuator signals. The latter is easy to generate by rapidly switching between two actuator levels, which can be done with current vibration exciters [1]. Other reasons for this choice are explained in Section 5

We apply our method to the Dynamic Darcy Cell experiment set-up, as detailed in [1, but we stress that our methodology can be applied to many other 
set-ups as well. The DOF in the Dynamic Darcy Cell set-up are the inlet volume and outlet volume. We introduce the Dynamic Darcy Cell in Section 2 and show how to apply sinusoidal and square wave signals to the set-up. Two types of measurements are then introduced: inlet pressure/outlet pressure measurements (Direct Method) and actuator position/outlet pressure measurements (Indirect Method). The former is one of the current ways to estimate parameters [1, in particular using sinusoidal signals. The latter has, to the authors' knowledge, not been investigated before. For both cases, we focus on square wave input signals, for which we prove that shorter experiment lengths than for sinusoidal ones can be obtained. We explain the data collection and estimation procedure in Section 3, and give an expression for the covariance matrix of the parameter estimates. In Sections 4 and 5 we use this expression to compute the optimal sinusoidal and square wave signals and DOF that minimize the experiment time for the estimation of permeability and porosity for the Direct and Indirect Methods. In the absence of a physical set-up, we illustrate the experiment design results by simulating the noise-corrupted physical system and applying the optimal square wave signal in Section 6. Lastly, we draw conclusions in Section 7.

\section{The Dynamic Darcy Cell}

To investigate the estimation problem the Dynamic Darcy Cell in [1] is considered. Detailed and schematic layouts of this set-up are depicted in Figs. 1 and 2. The porous core sample is mounted vertically in a core holder under a specific confined pressure $p_{\text {init }}$, with the inlet at the bottom (upstream side) and outlet at the top (downstream side). The sample with length $L$ and cross-sectional area $A$ is sealed at the top from the environment. The spatial coordinate axis $x$ is oriented towards the outlet and is perpendicular to the cross-sectional plane. The bottom of the sample is located at $x=0$. A vibration exciter with equilibrium position $x=-L_{i}$, also called the actuator, is moved according to the user-defined oscillatory signal $r(t)$. This signal is assumed to be continuous in 
time until Section 3, where instead discrete-time signals for estimation of the parameters are used. The actuator position $r$ as a function of time $t$ is either a sinusoid, i.e.,

$$
r(t)=C_{\gamma} \sin \left(\omega_{e x} t\right),
$$

or a square wave,

$$
r(t)=\frac{4}{\pi} C_{\gamma} \sum_{m=1}^{\infty} \frac{\sin \left([2 m-1] \omega_{c} t\right)}{2 m-1} .
$$

In these expressions, $\omega_{e x}$ and $\omega_{c}$ in respectively (1), (2) represent the excitation frequency of the sinusoid and cycle frequency (slowest mode) of the square wave. Furthermore, $C_{\gamma}$ is the amplitude of the actuator signal defined by

$$
C_{\gamma}=\gamma L_{i}, \quad 0<\gamma \leq \gamma_{m} .
$$

Obviously, the actuator amplitude can not exceed the length $L_{i}$ as can be seen from Fig. 2, so the user-chosen factor $\gamma$ has a geometric constraint $\gamma \leq \gamma_{m}=1$. However, the actuator amplitude may not necessarily be limited by the geometry of the setup but by its own movement restrictions, yielding a $\gamma_{m}$ that is smaller than unity.

The square wave signal can be generated in practice by switching abruptly between $+C_{\gamma}$ and $-C_{\gamma}$. The actuator induces an upstream (inlet) pressure signal at position $x=0$, i.e.,

$$
p_{i}(t)=p(x=0, t),
$$

within the inlet volume $V_{i}$ that is connected to the sample. This signal is measured by pressure transducer 1 (see Fig. 1) and assumed to be corrupted by sensor noise. At the sample outlet, which is connected to the end volume $V_{e}$, the output (downstream) pressure

$$
p_{e}(t)=y_{n f}(t)
$$

${ }_{138}$ is measured by pressure transducer 2 at $x=L$, see Fig. 1. Here, $y_{n f}(t)=$ ${ }_{39} p_{e}(t)=p(x=L, t)$ is the noise-free pressure response of the core sample. The 


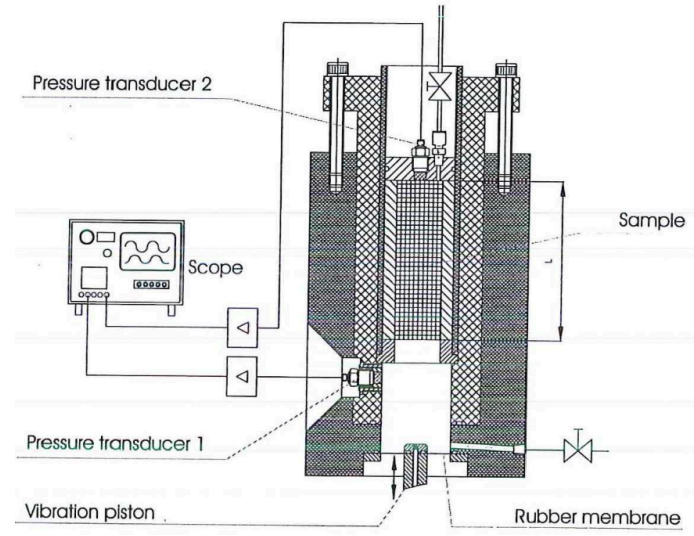

Figure 1: The Dynamic Darcy Cell. The core sample is mounted vertically in the holder. The actuator induces a pressure signal at inlet, measured by pressure transducer 1 . The wave propagates through the porous core and is measured at the outlet by pressure transducer 2 . Courtesy of [1].

input and output are continuous time signals. In Section 3 we will use sampled input and output data for parameter estimation. The measurements taken by this pressure transducer are also corrupted by sensor noise. We shall return to this point when we explain the estimation method in Section 3. The inlet and outlet volumes $V_{i}$ and $V_{e}$ can be set by the experimenter and are part of the DOF of the experiment set-up. (The set-up can for instance be designed to have easily-adaptable inlet and outlet volumes). Prior to the experiment the core, the inlet and outlet volume are filled with a gas (e.g., air) at pressure $p_{\text {init }}$.

\subsection{System of Coupled Equations in the Time Domain}

In the set-up we consider a porous cylindrical core that is homogeneous and isotropic with constant porosity $\phi$ and permeability $k$, and assume that the compressibility of the medium is small compared to the compressibility of the gas and therefore considered constant. Usually, both $\phi$ and $k$ have to be identified. The core is defined in Table 1. Gravity forces and pressure dependencies of the viscosity $\mu$ are neglected and isothermal conditions are assumed.

We now derive the dynamics within the experiment set-up. We shall consider 


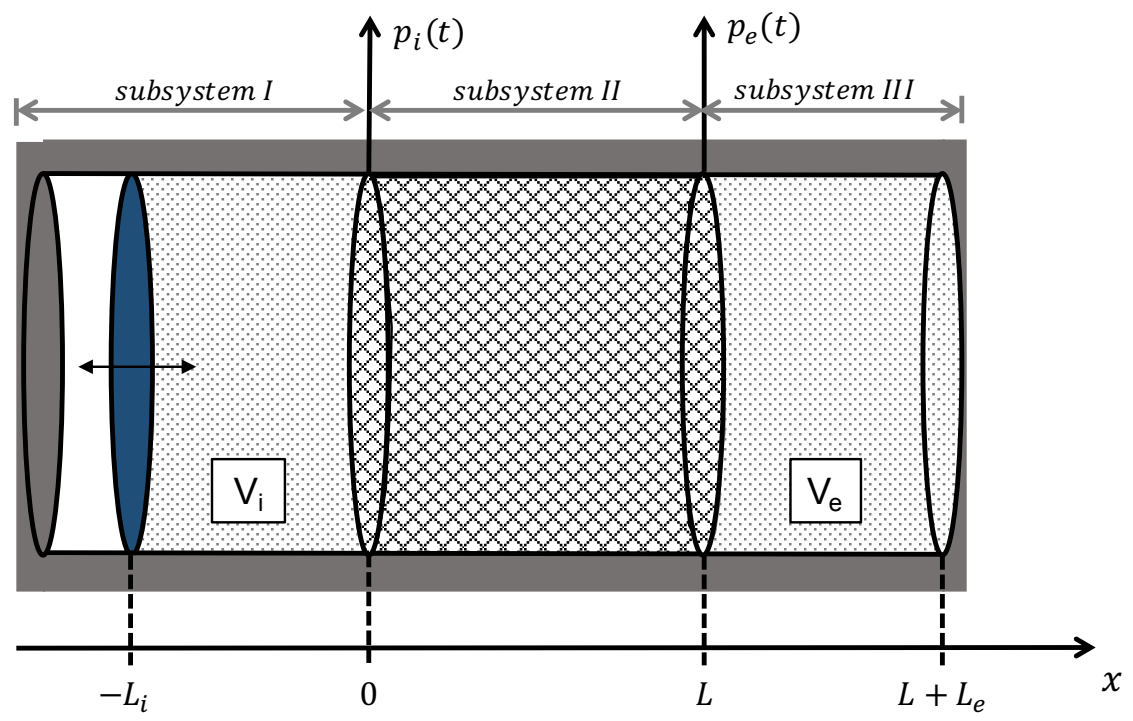

Figure 2: A sketch of the Dynamic Darcy Cell, rotated ninety degrees clock-wise. The cylindrical core sample is depicted in between the inlet volume $V_{i}$ and outlet volume $V_{e}$. The sample has a cross-sectional area $A$ and length $L$. The signals $p_{i}(t)$ and $p_{e}(t)$ are measured by pressure transducer 1 and 2 respectively, see Fig. 1 The actuator is visualised by the blue disk that can oscillate around its equilibrium position $x=-L_{i}$.

\begin{tabular}{l|l}
\hline Quantity & Value \\
\hline Length $L$ & $0.0512 \mathrm{~m}$ \\
Cross-sectional area $A$ & $\pi 0.0375^{2} / 4 \mathrm{~m}^{2}$ \\
Permeability $k_{0}$ & $2.0 \times 10^{-13} \mathrm{~m}^{2}$ \\
Porosity $\phi_{0}$ & 0.2 \\
Fluid density $\rho$ & $1.225 \mathrm{~kg} \mathrm{~m}^{3}$ \\
Dynamic Viscosity $\mu$ & $1.84 \times 10^{-5} \mathrm{~Pa} \mathrm{~s}$ \\
Bulk modulus $K$ & $1.0 \times 10^{5} \mathrm{~Pa}$ \\
\hline
\end{tabular}

Table 1: Parameters defining the Dynamic Darcy Cell and the core sample. These values define a core sample used in an actual pressure oscillation experiment in (1]). The parameters $k_{0}$ and $\phi_{0}$ need to be estimated. 
the inlet volume, the porous medium, and outlet volume as three subsystems with their own dynamics, input and output, and boundary conditions. The subsystems are interconnected at the inlet $(x=0)$ and outlet $(x=L)$ locations. We shall use the dynamics to derive the relationship between the inlet pressure to outlet pressure signals, and the actuator position to outlet pressure signals in Section 2.3 .

\section{Subsystem I: The Inlet Volume}

The actuator is a piston that vibrates in the $x$-direction within a confined gas in the inlet volume $V_{i}=A L_{i}$, where $L_{i}$ is the length of the inlet. The dynamics of the gas volume as a consequence of the moving piston is governed by the momentum and mass conservation laws, which for this particular configuration read

$$
\begin{aligned}
\frac{\partial w}{\partial t}+w \frac{\partial w}{\partial x}+\frac{1}{\rho} \frac{\partial p}{\partial x} & =0 \\
\frac{\partial \rho}{\partial t}+w \frac{\partial \rho}{\partial x}+\rho \frac{\partial w}{\partial x} & =0
\end{aligned}
$$

where $w=w(x, t)$ is the velocity profile of the gas in the $x$-direction (we use air as gas, see Table 1], $\rho$ the density of the gas, and $p=p(x, t)$ the pressure profile. We assume that the actuator has a small amplitude. In this case we may linearise (6)-(7) around the pressure $p_{i n i t}$. The factor $\gamma_{m}$ in $(3)$ is thus either defined by limitations in the actuator movement or by the linearisation condition, whichever is most restrictive. Furthermore, we assume isothermal conditions and therefore replace $\frac{\partial \rho}{\partial t}$ with $\frac{1}{K} \frac{\partial p}{\partial t}$, where $K=\rho \frac{\partial p}{\partial \rho}$ the Bulk modulus. Equations (6)-(7) then reduce to

$$
\begin{aligned}
\frac{\partial w}{\partial t}+\frac{1}{\rho} \frac{\partial p}{\partial x} & =0 \\
\frac{1}{K} \frac{\partial p}{\partial t}+\frac{\partial w}{\partial x} & =0
\end{aligned}
$$

where we furthermore neglected the nonlinear terms $w \frac{\partial w}{\partial x}$ and $w \frac{\partial \rho}{\partial x}$. Lastly, we take the derivative of (8) with respect to $x$ and the derivative of $(9)$ with respect 


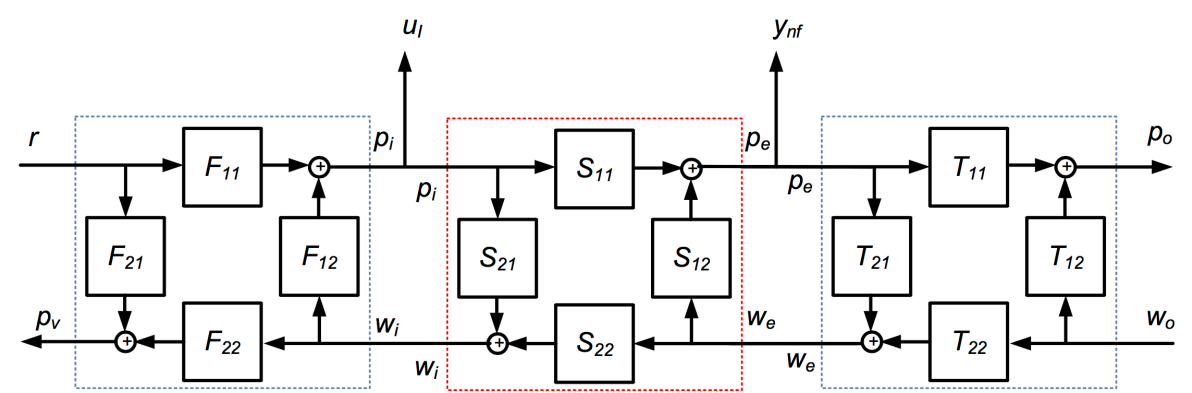

Figure 3: The network representation of the experimental setup. The dynamics inside the subsystems I and III are contained in respectively the left and right blue-dotted squares, whereas the dynamics in subsystem II is contained in the centre, red-dotted square.

to $t$ and combine the two, resulting in

$$
\begin{aligned}
\frac{\partial^{2} p}{\partial x^{2}} & =\frac{\rho}{K} \frac{\partial^{2} p}{\partial t^{2}}, \\
\frac{\partial w}{\partial t} & =-\frac{1}{\rho} \frac{\partial p}{\partial x} .
\end{aligned}
$$

The boundary conditions, i.e. inputs, of this subsystem are given by

$$
w\left(x=-L_{i}, t\right)=\frac{\partial r(t)}{\partial t}, w(x=0, t)=w_{i}(t)
$$

where $r(t)$ is the movement of the actuator as a function of time, and $w_{i}(t)$ the inlet gas speed. We neglect friction effects between the wall of the inlet volume and the actuator. The outputs of this subsystem are

$$
p\left(x=-L_{i}, t\right)=p_{v}(t), \quad p(x=0, t)=p_{i}(t) .
$$

This subsystem is shown in Fig. 3 in the left blue-dotted square, which reveals the coupling of all dynamics in the Laplace domain. Notice that it is connected to subsystem II through the output $p_{i}(t)$, being the inlet pressure, and the inlet gas speed $w_{i}(t)$. We now continue with the dynamics in the porous medium.

\section{Subsystem II: The Porous Medium}

For the second system, i.e. the porous medium, mass conservation and Darcy's law show that the pressure change in the core is governed by the diffusion 
equation

$$
\frac{\partial p(x, t)}{\partial t}=\frac{k K}{\mu \phi} \frac{\partial^{2} p(x, t)}{\partial x^{2}} .
$$

The specific discharge, or Darcy velocity, is related to pressure by

$$
w(x, t)=-\frac{k}{\mu} \frac{\partial p(x, t)}{\partial x} .
$$

Note that this velocity is different to those in the inlet and outlet volumes (subsystems I and III). The boundary conditions, i.e. inputs, for this subsystem read

$$
p(x=0, t)=p_{i}(t), w(x=L, t)=w_{e}(t),
$$

in which $p_{i}(t)$ is the inlet pressure defined previously, and $w_{e}(t)$ the outlet gas speed. The outputs are defined by

$$
w(x=0, t)=w_{i}(t), \quad p(x=L, t)=p_{e}(t) .
$$

This subsystem is shown in Fig. 3 in the centre, red-dotted square. Note that the first and second subsystem are now connected. What remains is to connect subsystem II with subsystem III. We shall now derive the dynamics in the latter.

\section{Subsystem III: The Outlet Volume}

In the third subsystem, following the same reasoning as for the first subsystem, the dynamics are determined by the conservation of mass and momentum. The pressure profile follows the wave equation and reads (c.f. 10p)

$$
\begin{aligned}
\frac{\partial^{2} p}{\partial x^{2}} & =\frac{\rho}{K} \frac{\partial^{2} p}{\partial t^{2}} \\
\frac{\partial w}{\partial t} & =-\frac{1}{\rho} \frac{\partial p}{\partial x} .
\end{aligned}
$$

The boundary conditions are however different from subsystem I and are given by

$$
p(x=L, t)=p_{e}(t), w\left(x=L+L_{e}, t\right)=w_{o}(t),
$$

in which $w_{o}(t)$ is an as-of-yet unspecified input for subsystem III. The outputs are defined by

$$
w(x=L, t)=w_{e}(t), \quad p\left(x=L+L_{e}, t\right)=p_{o}(t),
$$


where $w_{e}(t)$ is the gas speed at the outlet and $p_{o}(t)$ the pressure at the boundary $x=L+L_{e}$.

At this point, we have defined all dynamics in the experiment set-up. The three subsystems are now connected through their boundary conditions and outputs. Before proceeding with introducing the scaled set-up, we reflect on the assumptions made in the derivation of the governing equations in subsystems I until III.

\section{Range of Applicability}

We have assumed that (a) the flow is essentially laminar and inviscid in the inlet and outlet volumes, (b) that the flow in the core sample is laminar and non-inertial (Darcy-dominated), and (c) that temperature changes (and the associated changes in viscosity and density) of the working fluid are minimal, so that isothermal conditions can be assumed.

One way to test whether or not the flow is laminar in the inlet and outlet volume is to calculate the Reynolds number $\operatorname{Re}_{I, O}=\frac{\rho v L}{\mu}$, in which $v$ is the maximum flow velocity with units $[\mathrm{m} / \mathrm{s}], \rho$ the density of the fluid in $\left[\mathrm{kg} \mathrm{m}^{-3}\right]$, $L$ the characteristic travelled length of the fluid in [m], and $\mu$ the dynamic viscosity in $[\mathrm{Pa} \mathrm{s}]$. The subscript $I, O$ indicates that this definition of the Reynolds number pertains to the inlet and outlet volumes. If $\operatorname{Re}_{I, O} \leq 1$ the flow may be considered laminar. To validate assumption (b), one can utilise the following definition of the Reynolds number for porous media ([30]): $\operatorname{Re}=\frac{\rho w d}{\mu}$, where $\rho$ is again the density of the fluid, $w$ the specific discharge, and $d$ the typical grain diameter (which is roughly equivalent to the square root of permeability). If $\operatorname{Re}<10$ then the flow in the core is Darcy-dominated. An alternative method is to consider the Darcy-Forchheimer equation and compare the effect of the Darcy term with the inertial term; see $([31,[32])$. Anticipating low-frequency signals and the fact that usually water or air is used as gas in a Darcy experiment, (c) may be assumed to hold. For the core sample and excitation signals used in this work, the Reynolds numbers are $\operatorname{Re}_{I, O} \approx 0.8$ and $\operatorname{Re} \approx 0.03$, and thus within 
the accepted range.

We proceed by introducing the scaled set-up. The scaled equations are then used to find the dynamic relationship between the actuator, inlet pressure, and outlet pressure.

\subsection{Scaling}

We are interested to identify the porosity and the permeability of the porous medium. As shown in Table 1, the values of these parameters differ by twelve orders of magnitude. It is thus very important for the sake of numerical computations to scale the dynamical equations in such a way that the to-be-identified parameters are both of $O(1)$. To this end, we first choose $k_{s}$ and $\phi_{s}$ equal to the order of magnitude of $k$ and $\phi$ (which are usually known, either from prior experiment data, or for instance from the Rosetta Soil database from the US Salinity Lab [33]) and define the dimensionless parameters

$$
\tilde{k}=\frac{k}{k_{s}}, \quad \tilde{\phi}=\frac{\phi}{\phi_{s}} .
$$

${ }_{27}$ Furthermore, we also define the following dimensionless variables

$$
\tilde{x}=\frac{x}{L}, \tilde{p}(\tilde{x}, \tilde{t})=\frac{p\left(x / x_{s}, t / t_{s}\right)}{p_{s}}, \tilde{t}=\frac{t}{t_{s}}, \tilde{w}(\tilde{x}, \tilde{t})=\frac{w\left(x / x_{s}, t / t_{s}\right)}{w_{s}} .
$$

22 We note that the actuator signal $r(t)$ should be scaled in time and space accord229 ing to the above definitions, resulting in $\tilde{r}(\tilde{t})=\frac{r\left(t / t_{s}\right)}{L}$. The scaled frequency ${ }_{30} \tilde{\omega}$ follows from the above-defined time scaling and is given by $\tilde{\omega}=\omega t_{s}$. Lastly, choosing

$$
p_{s}=\frac{K}{\phi_{s}}, t_{s}=\frac{\phi_{s} \mu L^{2}}{k_{s} K}, w_{s}=\frac{k_{s} p_{s}}{\mu L}
$$


leads to equations with $O(1)$ terms when substituting $(22)-(24)$ into the equations of subsystem I to III 10-(18). The scaled system is thus given by

$$
\begin{aligned}
\frac{\partial^{2} \tilde{p}(\tilde{x}, \tilde{t})}{\partial \tilde{x}^{2}} & =\frac{\rho L^{2}}{K} t_{s}^{-2} \frac{\partial^{2} \tilde{p}(\tilde{x}, \tilde{t})}{\partial \tilde{t}^{2}}, \\
\frac{\partial \tilde{w}}{\partial \tilde{t}} & =-\frac{t_{s} p_{s}}{\rho w_{s} L} \frac{\partial \tilde{p}}{\partial \tilde{x}}, \\
\frac{\partial \tilde{p}(\tilde{x}, \tilde{t})}{\partial \tilde{t}} & =\frac{\tilde{k}}{\tilde{\phi}} \frac{\partial^{2} \tilde{p}(\tilde{x}, \tilde{t})}{\partial \tilde{x}^{2}} \\
\tilde{w}(\tilde{x}, \tilde{t}) & =-\tilde{k} \frac{\partial \tilde{p}(\tilde{x}, \tilde{t})}{\partial \tilde{x}}
\end{aligned}
$$

with the scaled boundary conditions $12,16,20$

$$
\begin{aligned}
\tilde{w}\left(\tilde{x}=-\frac{L_{i}}{L}, \tilde{t}\right) & =\frac{\partial \tilde{r}(\tilde{t})}{\partial \tilde{t}}, \tilde{w}(\tilde{x}=0, \tilde{t})=\tilde{w}_{i}(\tilde{t}), \\
\tilde{p}(\tilde{x}=0, \tilde{t}) & =\tilde{p}_{i}(\tilde{t}), \tilde{w}(\tilde{x}=1, \tilde{t})=\tilde{w}_{e}(\tilde{t}), \\
\tilde{p}(\tilde{x}=1, \tilde{t}) & =\tilde{p}_{e}(\tilde{t}), \tilde{w}\left(\tilde{x}=1+\frac{L_{e}}{L}, \tilde{t}\right)=\tilde{w}_{o}(\tilde{t}),
\end{aligned}
$$

and outputs 13, 17,21

$$
\begin{aligned}
\tilde{p}\left(\tilde{x}=-\frac{L_{i}}{L}, \tilde{t}\right) & =\tilde{p}_{v}(\tilde{t}), \quad \tilde{p}(\tilde{x}=0, \tilde{t})=\tilde{p}_{i}(\tilde{t}) \\
\tilde{w}(\tilde{x}=0, \tilde{t}) & =\tilde{w}_{i}(\tilde{t}), \quad \tilde{p}(\tilde{x}=1, \tilde{t})=\tilde{p}_{e}(\tilde{t}) \\
\tilde{w}(\tilde{x}=1, \tilde{t}) & =\tilde{w}_{e}(\tilde{t}), \tilde{p}\left(\tilde{x}=1+\frac{L_{e}}{L}, \tilde{t}\right)=\tilde{p}_{o}(\tilde{t}) .
\end{aligned}
$$

From now on we shall work with the scaled system and omit the tildes for the sake of simplicity. The unscaled results can easily be deduced using the scaling defined in this section. We proceed with the determination of the dynamic relationships between the actuator and the inlet and outlet pressures.

\subsection{System of Coupled Equations in the Laplace Domain}

For the estimation of the physical parameters we require explicit expressions of the transfer functions between the actuator and the outlet pressure, as well as the inlet to outlet pressure. For this purpose, we transform the equations for each system into the Laplace domain. 
The dynamics in subsystem I are defined by $10-(13)$. Their scaled equivalents are given by $(25)-(26),(29)$, and $(32)$. We take the Laplace transform of 25 and assume zero initial conditions. The resulting equation reads

$$
\frac{\partial^{2} P(x, s)}{\partial x^{2}}=\frac{\rho L^{2} s^{2}}{K t_{s}^{2}} P(x, s)
$$

where $P(x, s)$ is the Laplace transform of the scaled pressure profile $p(x, t)$. This equation has the general solution

$$
P(x, s)=m_{1} \sinh \left(x \frac{L}{t_{s}} \sqrt{\frac{\rho}{K}} s\right)+m_{2} \cosh \left(x \frac{L}{t_{s}} \sqrt{\frac{\rho}{K}} s\right),
$$

in which $m_{1}$ and $m_{2}$ are two unknown scalars. We solve the coefficients $m_{1}, m_{2}$ with the equation for the gas speed $(26)$, which we transform into the Laplace domain (assuming zero initial conditions), giving

$$
W(x, s)=-\frac{t_{s} p_{s}}{\rho w_{s} L s} \frac{\partial P(x, s)}{\partial x} .
$$

Substitution of 36 into 37 then yields

$$
W(x, s)=-\frac{p_{s}}{w_{s} \sqrt{\rho K}}\left\{m_{1} \cosh \left(x \frac{L}{t_{s}} \sqrt{\frac{\rho}{K}} s\right)+m_{2} \sinh \left(x \frac{L}{t_{s}} \sqrt{\frac{\rho}{K}} s\right)\right\} .
$$

Using the Laplace transformed boundary conditions 29 then leads to

$$
\begin{aligned}
& m_{1}=-\frac{w_{s}}{p_{s}} \sqrt{\rho K} W_{i}(s) \\
& m_{2}=-\frac{w_{s}}{p_{s}} \sqrt{\rho K}\left\{\sinh ^{-1}\left(-s \frac{L_{i}}{t_{s}} \sqrt{\frac{\rho}{K}}\right) s R(s)-\operatorname{coth}\left(-s \frac{L_{i}}{t_{s}} \sqrt{\frac{\rho}{K}}\right) W_{i}(s(4))\right.
\end{aligned}
$$

Substitution of these expressions into 36 and using the Laplace transformed outputs 32 allows us to determine the dynamical relationship between $R(s)$, $W_{e}(s)$ and $P_{v}(s), P_{i}(s)$ :

$$
\left[\begin{array}{c}
P_{i}(s) \\
P_{v}(s)
\end{array}\right]=\frac{w_{s}}{p_{s}} \sqrt{\rho K}\left[\begin{array}{ll}
F_{11}(s) & F_{12}(s) \\
F_{21}(s) & F_{22}(s)
\end{array}\right]\left[\begin{array}{l}
R(s) \\
W_{i}(s)
\end{array}\right],
$$

in which

$$
\left[\begin{array}{cc}
F_{11}(s) & F_{12}(s) \\
F_{21}(s) & F_{22}(s)
\end{array}\right]=\left[\begin{array}{cc}
-s \sinh ^{-1}\left(-s \frac{L_{i}}{t_{s}} \sqrt{\frac{\rho}{K}}\right) & \operatorname{coth}\left(-s \frac{L_{i}}{t_{s}} \sqrt{\frac{\rho}{K}}\right) \\
-s \operatorname{coth}\left(-s \frac{L_{i}}{t_{s}} \sqrt{\frac{\rho}{K}}\right) & \sinh ^{-1}\left(-s \frac{L_{i}}{t_{s}} \sqrt{\frac{\rho}{K}}\right)
\end{array}\right] .
$$


The argument in each of the above hyperbolic functions contains the fraction $\frac{L_{i} \sqrt{\rho}}{t_{s} \sqrt{K}}=\frac{k_{s} L_{i} \sqrt{\rho K}}{L^{2} \mu \phi_{s}}$. Since this expression evaluates to a small number for typical values of each parameter, we approximate these hyperbolic functions with $\operatorname{coth}(z) \approx \frac{1}{z}$ and $\sinh (z) \approx z$ where $z \ll 1$. The transfer functions then reduce to the expressions

$$
\left[\begin{array}{ll}
F_{11}(s) & F_{12}(s) \\
F_{21}(s) & F_{22}(s)
\end{array}\right]=\frac{\phi_{s} L}{L_{i} s}\left[\begin{array}{cc}
s & -1 \\
s & -1
\end{array}\right]=\frac{f_{i}}{s}\left[\begin{array}{cc}
s & -1 \\
s & -1
\end{array}\right],
$$

where $f_{i}$ is defined as

$$
f_{i}=\frac{\phi_{s} A L}{V_{i}}=\frac{\phi_{s} L}{L_{i}}
$$

\section{Subsystem II}

We recall that the dynamics in subsystem II are defined by the equations (14)-(17). Their scaled equivalents are given by (27)-228, (30), and (33). We apply the Laplace transform to the diffusion equation (27), again assuming zero initial conditions, and write it as

$$
\left(\frac{\partial^{2}}{\partial x^{2}}-\frac{s}{k / \phi}\right) P(x, s)=0
$$

where $P(x, s)$ is the Laplace transformed scaled pressure. The general solution to this equation reads

$$
P(x, s)=c_{1} e^{x \sqrt{\frac{s \phi}{k}}}+c_{2} e^{-x \sqrt{\frac{s \phi}{k}}},
$$

where $c_{1}$ and $c_{2}$ are as-of-yet unknown scalar coefficients. Taking the Laplace transform of 28) and using the previous equation shows that the speed of gas in the porous medium is given by

$$
W(x, s)=-k c_{1} \sqrt{\frac{s \phi}{k}} e^{x \sqrt{\frac{s \phi}{k}}}+k c_{2} \sqrt{\frac{s \phi}{k}} e^{-x \sqrt{\frac{s \phi}{k}}} .
$$

The unknown coefficients $c_{1}$ and $c_{2}$ are then determined with the Laplace transformed boundary conditions (30). We then substitute these into (46) and use (33) to find the dynamical relationships between $P_{e}(s), W_{i}(s)$ and $P_{i}(s), W_{e}(s)$ :

$$
\left[\begin{array}{c}
P_{e}(s) \\
W_{i}(s)
\end{array}\right]=\left[\begin{array}{ll}
S_{11}(s) & S_{12}(s) \\
S_{21}(s) & S_{22}(s)
\end{array}\right]\left[\begin{array}{c}
P_{i}(s) \\
W_{e}(s)
\end{array}\right]
$$


in which

$$
\left[\begin{array}{cc}
S_{11}(s) & S_{12}(s) \\
S_{21}(s) & S_{22}(s)
\end{array}\right]=\left[\begin{array}{cc}
\cosh ^{-1}(\sqrt{s \phi / k}) & \frac{-1}{\sqrt{s \phi k}} \tanh (\sqrt{s \phi / k}) \\
\sqrt{s \phi k} \tanh (\sqrt{s \phi / k}) & \cosh ^{-1}(\sqrt{s \phi / k})
\end{array}\right] .
$$

\section{Subsystem III}

The dynamics in subsystem III are defined by the equations (18)-21). Their scaled equivalents are given by (25)-(26), 31), and (34). We follow the exact same derivation as for subsystem I, albeit with the boundary conditions and outputs in (31) and (34). The result for subsystem III is that

$$
\left[\begin{array}{c}
P_{o}(s) \\
W_{e}(s)
\end{array}\right]=\left[\begin{array}{cc}
T_{11}(s) & T_{12}(s) \\
T_{21}(s) & T_{22}(s)
\end{array}\right]\left[\begin{array}{c}
P_{e}(s) \\
W_{o}(s)
\end{array}\right]
$$

in which

$$
\left[\begin{array}{cc}
T_{11}(s) & T_{12}(s) \\
T_{21}(s) & T_{22}(s)
\end{array}\right]=\left[\begin{array}{cc}
\cosh ^{-1}\left(\frac{L_{e}}{t_{s}} \sqrt{\frac{\rho}{K}} s\right) & -\frac{w_{s}}{p_{s}} \sqrt{\rho K} \tanh \left(\frac{L_{e}}{t_{s}} \sqrt{\frac{\rho}{K}} s\right) \\
\frac{p_{s}}{w_{s} \sqrt{\rho K}} \tanh \left(\frac{L_{e}}{t_{s}} \sqrt{\frac{\rho}{K}} s\right) & \cosh ^{-1}\left(\frac{L_{e}}{t_{s}} \sqrt{\frac{\rho}{K}} s\right)
\end{array}\right] .
$$

Following the derivation for subsystem I we remark that the arguments in all the hyperbolic functions are small. We approximate these functions around zero and find that we may write

$$
\left[\begin{array}{ll}
T_{11}(s) & T_{12}(s) \\
T_{21}(s) & T_{22}(s)
\end{array}\right]=\left[\begin{array}{cc}
1 & -\frac{k_{s}^{2} K \rho}{\mu^{2} L^{2}} \frac{s}{f_{e}} \\
\frac{s}{f_{e}} & 1
\end{array}\right],
$$

where $f_{e}$ is defined as

$$
f_{e}=\frac{\phi_{s} A L}{V_{e}}=\frac{\phi_{s} L}{L_{e}} .
$$

\section{The Experiment Set-Up System}

At this point we have coupled the different inputs and outputs of the total system. In the Dynamic Darcy Cell experiment set-up, however, we do not have gas entering the system at $x=1+\frac{L_{e}}{L}$ and thus $w_{o}(t)=0$. Consequently, we 


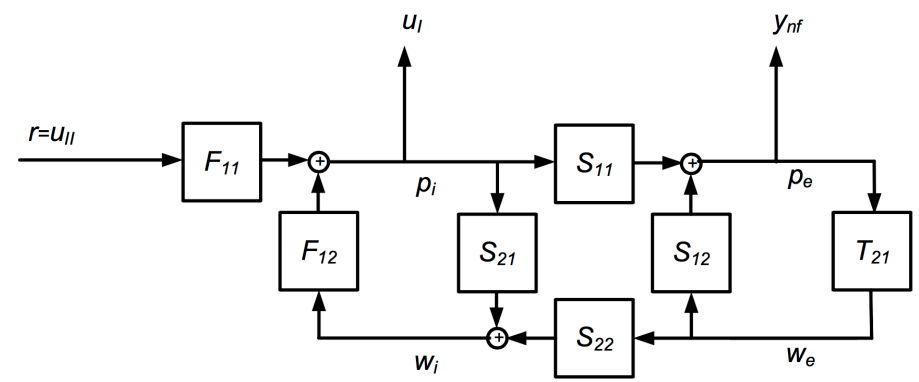

Figure 4: The network representation of the experimental setup after simplification. $270 \quad 4$

We start with the relationship between $P_{i}$ and $P_{e}$, which we shall use to find the time-domain connection between $p_{e}(t)$ and $p_{i}(t)$. We find from Fig. 4 that

$$
P_{e}(s)=\frac{S_{11}(s)}{1-S_{12}(s) T_{21}(s)} P_{i}(s) .
$$

This equation shows that the outlet pressure depends on both the inlet pressure $P_{i}$ as well as itself through $W_{e}$. This feedback mechanism is clearly seen in Fig.

Next, the relationship between $R(s)$ and $P_{i}(s)$ can be deduced from 443, 
(48)-(49), and (50) or Fig. 4 and reads

$$
P_{i}(s)=F_{11}(s)\left[1-F_{12}(s)\left(S_{21}(s)+S_{22}(s) T_{21}(s) \frac{S_{11}(s)}{1-S_{12}(s) T_{21}(s)}\right)\right]^{-1} R(s) .
$$

Equations (54) and (55) reveal the transfer functions between the Laplacetransformed measurement variables $r(t), p_{i}(t)$, and $p_{e}(t)$. In the next section we show how to calculate the time-domain response of $p_{i}(t)$ and $p_{e}(t)$ as a function of the actuator signal $r(t)$ using these transfer functions. These responses are a function of the physical parameters that we seek to estimate, and will be used as estimation functions in Section 3 .

\subsection{Input-Output Dynamics in the Time Domain}

Suppose that an input signal $u(t)$ (which in our case can either be $p_{i}(t)$ or $r(t))$ affects some noise-free output $y_{n f}(\boldsymbol{\theta}, t)$. In the Laplace domain, their relation is

$$
Y_{n f}(\boldsymbol{\theta}, s)=G(s, \boldsymbol{\theta}) U(s),
$$

where $G(s, \boldsymbol{\theta})$ is a function of the physical parameters collected in the vector $\boldsymbol{\theta}$. This vector represents a family of physical systems, whereas $\boldsymbol{\theta}=\boldsymbol{\theta}_{0}$ refers to a particular physical system characterised by its parameters $\boldsymbol{\theta}_{0}$. We shall refer to the system with $\boldsymbol{\theta}_{0}$ as the data-generating system, and $\boldsymbol{\theta}_{0}$ contains the physical parameters we need to estimate. As will become clear in Section 3 . we shall use discrete-time input and output data to estimate the parameters using a least-squares method. To this end, we require a function that simulates the noise-free response $y_{n f}(\boldsymbol{\theta}, t)$ of the system. In this section, we derive this response for sinusoidal (1) and square wave (2) actuator signals.

Suppose we have a closed-form expression of $G(s, \boldsymbol{\theta})$ in 556 . The timedomain signal $y_{n f}(\boldsymbol{\theta}, t)$ for an input signal of the form, assuming zero initial conditions, reads

$$
u(t)=C_{\gamma} \sin (\omega t) .
$$


The corresponding noise-free output is then given by 28 ]

$$
y_{n f}(\boldsymbol{\theta}, t)=C_{\gamma}|G(i \omega, \boldsymbol{\theta})| \sin (\omega t+\alpha),
$$

where $G(i \omega, \boldsymbol{\theta})$ is defined in 56 and $\alpha=\angle G(i \omega, \boldsymbol{\theta})$. In other words, the output $y_{n f}(\boldsymbol{\theta}, t)$ is also a sinusoid with frequency $\omega$ but a different amplitude and phase. Using this insight, it is straightforward to write down an expression for the output when the input is a sum of sinusoids. Indeed, for a square wave input signal (2) with amplitude $C$ and cycle frequency $\omega_{c}$ the output $y_{n f}(\boldsymbol{\theta}, t)$ reads

$$
y_{n f}(\boldsymbol{\theta}, t)=\frac{4}{\pi} C_{\gamma} \sum_{m=1}^{\infty} \frac{\left|G\left(i[2 m-1] \omega_{c}, \boldsymbol{\theta}\right)\right|}{2 m-1} \sin \left([2 m-1] \omega_{c} t+\alpha_{m}\right),
$$

where $\alpha_{m}=\angle G\left(i[2 m-1] \omega_{c}, \boldsymbol{\theta}\right)$. This is an elegant way to find the timedomain expression of the output of the system without requiring an inverse Laplace transform.

At this point a general expression of a linear system's response for sinusoidal and square wave input signals is defined. What remains is to find a closed-form expression for the transfer function $G(s, \boldsymbol{\theta})$ that connects the actuator signal $r(t)$ with the inlet and outlet pressures $p_{i}(t)$ and $p_{e}(t)$. In the next two subsections we derive this transfer function for $p_{i}(t)$ to $p_{e}(t)$ and $r(t)$ to $p_{e}(t)$.

Direct Method: Inlet-Outlet Pressure Measurements

The first measurement method uses inlet and outlet pressure measurements. This is one way to collect data in pressure oscillation experiments, see e.g. [1. The input signal $u(t)=u_{I}(t)=p_{i}(t)$ and the output is $y_{n f}(\boldsymbol{\theta}, t)=p_{e}(t)$, see Fig. 4. In the previous section we have derived the relationship between $P_{i}(s)$ and $Y_{n f}(s)=P_{e}(s)$ (c.f. (54)), being

$$
P_{e}(s)=\frac{S_{11}}{1-S_{12} T_{21}} P_{i}(s) .
$$

Substitution of $S_{11}, S_{12}$, and $T_{21}$ defined in $(49)$ and (50) results in

$$
P_{e}(s)=G_{f_{e}}(s, \boldsymbol{\theta}) P_{i}(s),
$$


in which

$$
G_{f_{e}}(s, \boldsymbol{\theta})=\frac{1}{\cosh \sqrt{\phi s / k}+f_{e}^{-1} \sqrt{s(\phi k)^{-1}} \sinh \sqrt{\phi s / k}} .
$$

Note that $\boldsymbol{\theta}=[k, \phi]^{T}$ and that $G_{f_{e}}$ also depends on the degree of freedom $f_{e}$. We have now obtained the closed-form expression of the transfer function between $p_{i}(t)$ and $p_{e}(t)$. Hence, we can easily calculate the output response in the time domain using (58). We remark that the dynamics between $p_{i}(t)$ and $p_{e}(t)$ have also been derived in [1] but that the concept of transfer function has not been used, see Appendix C for a discussion.

For future reference we also calculate the gradient of 62 with respect to $\boldsymbol{\theta}=(k, \phi)$ :

$$
\begin{aligned}
& \frac{\partial G_{f_{e}}(i \omega, \boldsymbol{\theta})}{\partial k}=\frac{f_{e}\left\{i \phi \omega \cosh \left(\sqrt{\frac{i \omega \phi}{k}}\right)+k\left[f_{e} \phi+1\right] \sqrt{\frac{i \omega \phi}{k}} \sinh \left(\sqrt{\frac{i \omega \phi}{k}}\right)\right\}}{2 k^{2} \phi\left\{f_{e} \cosh \left(\sqrt{\frac{i \omega \phi}{k}}\right)+\sqrt{i \omega(k \phi)^{-1}} \sinh \left(\sqrt{\frac{i \omega \phi}{k}}\right)\right\}^{2}} \\
& \frac{\partial G_{f_{e}}(i \omega, \boldsymbol{\theta})}{\partial \phi}=-\frac{f_{e}\left\{i \omega / k \cosh \left(\sqrt{\frac{i \omega \phi}{k}}\right)+\left[f_{e} \sqrt{\frac{i \omega \phi}{k}}-\sqrt{i \omega(k \phi)^{-1}}\right] \sinh \left(\sqrt{\frac{i \omega \phi}{k}}\right)\right\}_{4)}}{2 \phi\left\{f_{e} \cosh \left(\sqrt{\frac{i \omega \phi}{k}}\right)+\sqrt{i \omega(\phi k)^{-1}} \sinh \left(\sqrt{\frac{i \omega \phi}{k}}\right)\right\}^{2}}
\end{aligned}
$$

We shall use these expressions in Section 4 to find the optimal input signals. The absolute values of the derivatives are shown in Figs. 5 and 6 as a function of $\omega$ for several values of $f_{e}$.

\section{Indirect Method: Actuator Position/Outlet Pressure Measurements}

Another possible method uses the actuator and outlet pressure signal for parameter estimation. These signals have so far not been considered in literature for identification, but have a major advantage with respect to the Direct Method. Indeed, since the actuator signal is applied and not measured, no sensor noise is present on the actuator signal. Therefore, it is not an Errors-in-Variables problem as the Direct Method is. For the Indirect Method, the input and output are respectively given by $u(t)=u_{I I}(t)=r(t)$ and $y_{n f}(\boldsymbol{\theta}, t)=p_{e}(t)$, see 


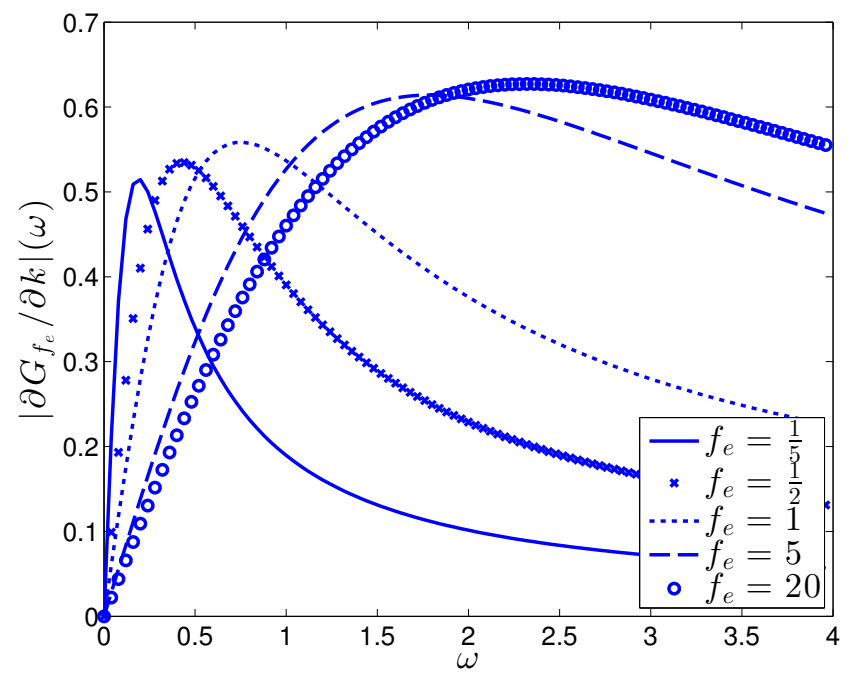

Figure 5: Absolute value of the derivative of the transfer function 62 with respect to permeability as a function of the scaled frequency $\omega$, see 63 . Observe that for increasing values of $f_{e}$, the maximum of the curve shifts to higher frequencies and higher values. A small (large) $f_{e}$ value means that the outlet volume is large (small).

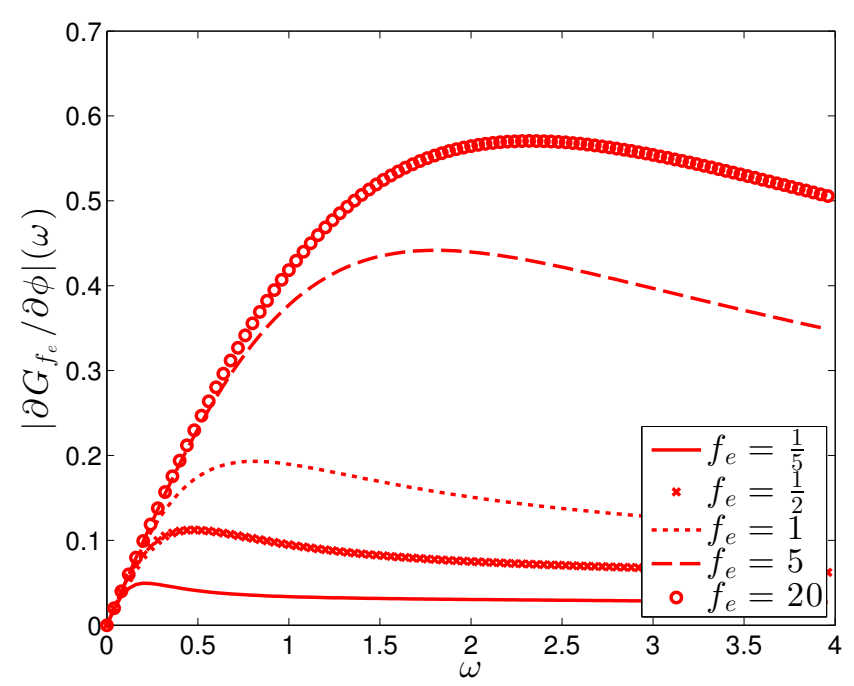

Figure 6: Absolute value of the derivative of the transfer function 62 with respect to porosity as a function of the scaled frequency $\omega$, see 64 . For a given ratio $f_{e}$, the absolute value of $\partial G_{f_{e}}(i \omega, \boldsymbol{\theta}) / \partial \phi$ is smaller at almost all frequencies than the absolute value of $\partial G_{f_{e}}(i \omega, \boldsymbol{\theta}) / \partial k$, see Figure 5 
Fig. 4. The relationship between $r(t)$ and $p_{e}(t)$ in the Laplace domain is found by combining (54), 555) and using (62), i.e.,

$$
P_{e}(s)=\left[1-F_{12}\left(S_{21}+S_{22} T_{21} G_{f_{e}}(s, \boldsymbol{\theta})\right)\right]^{-1} F_{11}(s) G_{f_{e}}(s, \boldsymbol{\theta}) R(s)
$$

Substitution of $F_{11}, F_{12}$ (43), $S_{11}, \ldots, S_{22}$ 497, and $T_{21}$ (50), into the previous expression results in

$$
P_{e}(s)=L_{f_{i}, f_{e}}(s, \boldsymbol{\theta}) G_{f_{e}}(s, \boldsymbol{\theta}) R(s),
$$

in which

$$
L_{f_{i}, f_{e}}(s, \boldsymbol{\theta})=\left[\frac{1}{f_{i}}+\frac{1}{s}\left(k \sqrt{\frac{s \phi}{k}} \tanh \sqrt{\frac{s \phi}{k}}+\frac{s G_{f_{e}}(s, \boldsymbol{\theta})}{f_{e} \cosh \sqrt{\frac{s \phi}{k}}}\right)\right]^{-1} .
$$

This is an interesting result. It shows that the relationship between $R(s)$ and $P_{e}(s)$ is equal to the relationship between $P_{i}(s)$ and $P_{e}(s)$ multiplied by a complex filter $L_{f_{i}, f_{e}}(s, \boldsymbol{\theta})$. Thus, we have that $P_{i}(s)=L_{f_{i}, f_{e}}(s) R(s)$ and $P_{e}(s)=G_{f_{e}}(s) P_{i}(s)$. The input-output connection between $R(s)$ and $P_{e}(s)$ is thus $P_{e}(s)=L_{f_{i}, f_{e}}(s, \boldsymbol{\theta}) G_{f_{e}}(s, \boldsymbol{\theta}) R(s)=G_{f_{i}, f_{e}}(s, \boldsymbol{\theta}) R(s)$, where

$$
G_{f_{i}, f_{e}}(s, \boldsymbol{\theta})=\left[\frac{1}{f_{i}}+\frac{1}{s}\left(k \sqrt{\frac{s \phi}{k}} \tanh \sqrt{\frac{s \phi}{k}}+\frac{s G_{f_{e}}(s, \boldsymbol{\theta})}{f_{e} \cosh \sqrt{\frac{s \phi}{k}}}\right)\right]^{-1} G_{f_{e}}(s, \boldsymbol{\theta}) .
$$

For future reference, we also calculate the derivatives of 68 with respect to $k$ and $\phi$ :

$$
\begin{aligned}
& k \phi G_{f_{e}}(i \omega, \boldsymbol{\theta})\left[\sqrt{\frac{i \omega \phi}{k}} \operatorname{sech}^{2} \sqrt{\frac{i \omega \phi}{k}}-\tanh \sqrt{\frac{i \omega \phi}{k}}\right]-i f_{e}^{-1} \phi \omega G_{f_{e}}^{2}(i \omega, \boldsymbol{\theta}) \\
& \frac{\partial G_{f_{i}, f_{e}}(i \omega, \boldsymbol{\theta})}{\partial k}=\frac{\times \operatorname{sech} \sqrt{\frac{i \omega \phi}{k}} \tanh \sqrt{\frac{i \omega \phi}{k}}+2 f_{i}^{-1} k^{2} \frac{\partial G_{f_{e}}(i \omega, \boldsymbol{\theta})}{\partial k}\left[\sqrt{\frac{i \omega \phi}{k}}+f_{i} \phi \tanh \sqrt{\frac{i \omega \phi}{k}}\right]_{(69)}}{2 k^{2} \sqrt{\frac{i \omega \phi}{k}}\left(f_{i}^{-1}+f_{e}^{-1} G_{f_{e}}(i \omega, \boldsymbol{\theta}) \operatorname{sech} \sqrt{\frac{i \omega \phi}{k}}+\phi \sqrt{\frac{k}{i \omega \phi}} \tanh \sqrt{\frac{i \omega \phi}{k}}\right)^{2}} \\
& i \phi \omega\left(\sqrt{k} G_{f_{e}}(i \omega, \boldsymbol{\theta}) \operatorname{sech}^{2} \sqrt{\frac{i \omega \phi}{k}}-2 f_{i}^{-1} \sqrt{k} \frac{\partial G_{f_{e}}(i \omega, \boldsymbol{\theta})}{\partial \phi}+\frac{\tanh \sqrt{\frac{i \omega \phi}{k}}}{\sqrt{i \omega \phi}}\right.
\end{aligned}
$$

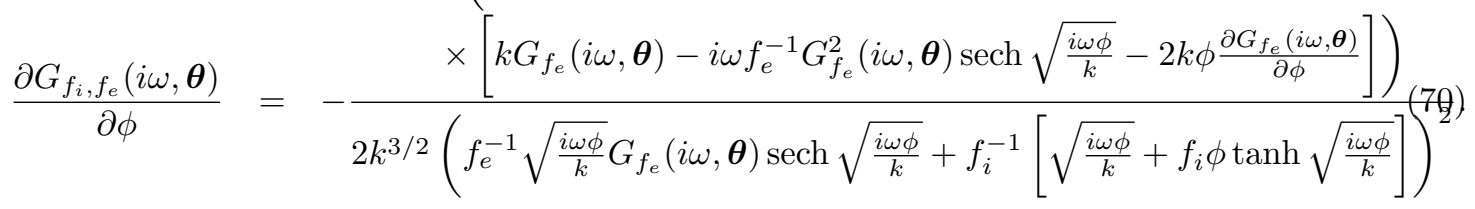




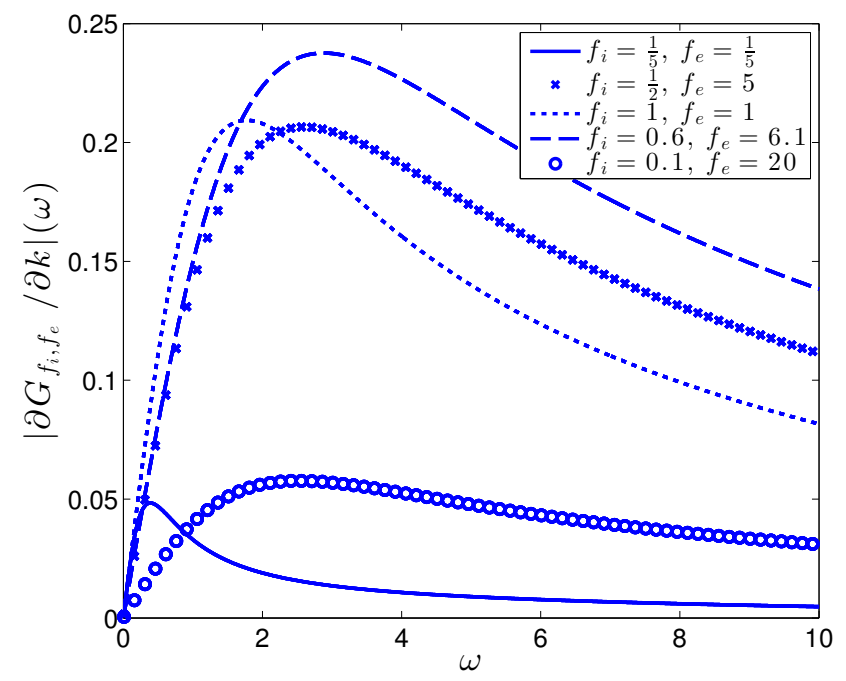

Figure 7: Absolute value of the derivative of the transfer function 68 with respect to permeability as a function of the scaled frequency $\omega$, see 69]. Observe that for increasing values of $f_{i}$ and $f_{e}$, the maximum of the curve increases and shifts to higher frequencies. A small (large) $f_{e}$ value means that the outlet volume is large (small). A small (large) $f_{i}$ value means that the inlet volume is large (small).

We first rewrite the expression of the actuator amplitude $C_{\gamma}$ (c.f. (3)). Since $C_{\gamma}$ and the ratio $f_{i}(44)$ are related to the inlet length $L_{i}$, we write the actuator amplitude as a function of $f_{i}$, i.e.

$$
C_{\gamma}\left(f_{i}\right)=\gamma \frac{L_{i}}{L}=\gamma \frac{\phi_{s}}{f_{i}}
$$




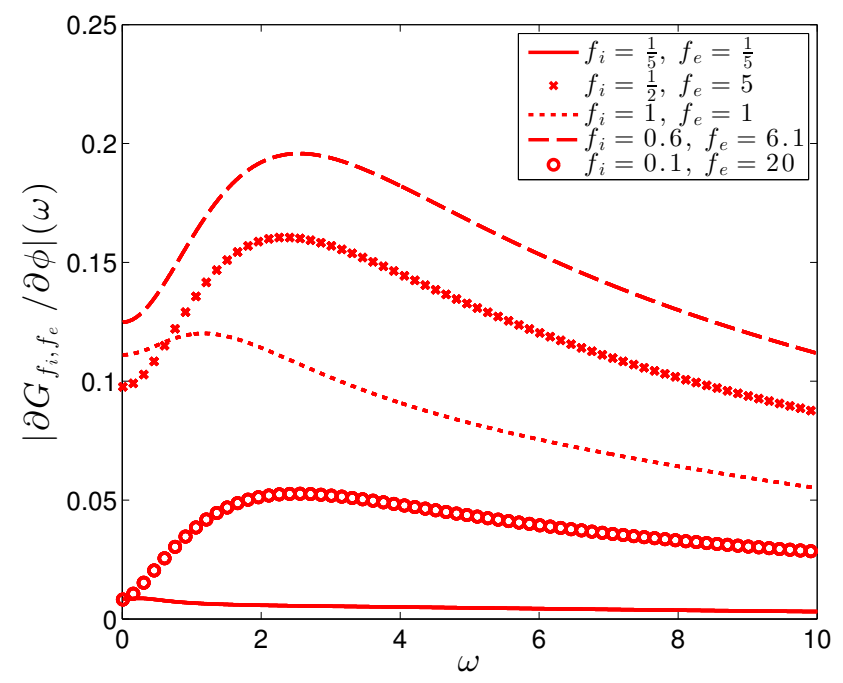

Figure 8: Absolute value of the derivative of the transfer function 68 with respect to porosity as a function of the scaled frequency $\omega$, see 70 .

where we still have that $0<\gamma \leq \gamma_{m}$. It is already mentioned that $\gamma_{m} \leq 1$ due to the movement limitations of the actuator. However, it is now also clear that $\gamma_{m}$ can not be chosen large as otherwise the inlet volume is no longer approximately constant due to the actuator movement, and hence our above derivations no longer hold. It is important to use the this definition of the actuator amplitude as otherwise unphysical (wrong) results are obtained when using optimal experiment design. The value $\gamma_{m} \leq 1$ should be determined by the experimenter.

We continue with determining the data collection procedure and then define the least-squares method. In the remainder of this article we derive results based on a square wave actuator signal (with an as-of-yet undetermined amplitude and cycle frequency), which we prove in Appendix D to deliver shorter experiment lengths than a sinusoidal one for the same values of $f_{i}$ and $f_{e}$. 


\section{Indirect Method}

Let us for simplicity first explain the identification of the parameters for the Indirect Method. In system identification, parameters of a system are estimated based on input-output data collected on the system. For the Indirect Method we already mentioned that the input signal for the identification is a discretetime version of the user-chosen actuator signal $r(t)$ and the output signal is a discrete-time version of the outlet pressure $p_{e}(t)$. An identification experiment can be performed as follows. We apply, for a certain duration, a continuous-time excitation signal $r(t)$ of the form (2) and we wait for the transients to die out. The corresponding steady-state output signal (i.e the outlet pressure) is then measured at a sampling rate $T_{s}$. We will suppose that an anti-aliasing filter is applied prior to the sampling. In this way, we are able to collect $N$ output measurements $y_{D}[n](n=1, \ldots, N)$ with $N T_{s}$ the duration of the experiment. These measurements are of course corrupted by noise. In the sequel, we suppose that this noise is white and has a variance $\sigma_{e}^{2}$. Coloured noise could also be taken into account, see e.g. 28. The continuous-time input signal $r(t)$ also has to be transformed into a discrete-time signal that we will denote by $u_{D}[n]$. This discretization is done in the same way as for the output signal. First, the signal is filtered by an anti-aliasing filter and then sampled. Since we precisely know $r(t)$, we can exactly compute $u_{D}[n](n=1, \ldots, N)$. This leads to the following input data $(n=1, \ldots, N)$ :

$$
u_{D}[n]=\frac{4}{\pi} C_{\gamma}\left(f_{i}\right) \sum_{m=1}^{Q\left(T_{s}\right)} \frac{\sin \left([2 m-1] \omega_{c} n T_{s}\right)}{2 m-1},
$$

where we recall that $\omega_{c}$ is the cycle frequency of the square wave. Furthermore, observe that the sum no longer extends to infinity but to the finite integer $Q\left(T_{s}\right)=\left\lfloor\frac{1}{2}\left(\frac{\pi}{\omega_{c} T_{s}}+1\right)\right\rfloor:$ it determines the highest mode that can be observed in the discrete-time data. Here, $\lfloor\cdot\rfloor$ represents the floor function. This integer can be easily deduced from the radial Nyquist rate $\frac{\pi}{T_{s}}$.

Using this procedure, we have obtained an input-output data set $Z_{N}=\left\{u_{D}[n], y_{D}[n]\right\}_{n=1, \ldots, N}$ with which the unknown parameter vector $\boldsymbol{\theta}_{0}=$ 
$\left(k_{0}, \phi_{0}\right)$ will be estimated. In order to determine an optimal estimator for this purpose, we need to write an expression of the output data as a function of variables that represent these parameters. This can be easily done. Indeed, due to our data generating mechanism, in the frequency range $\left[0, \pi / T_{s}\right]$, the frequency response $G_{d}\left(e^{i \omega T_{s}}, \boldsymbol{\theta}_{0}\right)$ of the discrete-time transfer function $G_{d}\left(z, \boldsymbol{\theta}_{0}\right)$ between the input data $u_{D}[n]$ and the (noise-free) output data $y_{n f}[n]$ is perfectly equal to the frequency response $G_{f_{i}, f_{e}}\left(i \omega, \boldsymbol{\theta}_{0}\right)$ of the continuous-time transfer function $G_{f_{i}, f_{e}}\left(s, \boldsymbol{\theta}_{0}\right)$ between the continuous-time input and output. Consequently, we can write

$$
y_{D}[n]=y_{n f}\left(\boldsymbol{\theta}=\boldsymbol{\theta}_{0}\right)[n]+e[n],
$$

where $e[n]$ is a white noise realisation with variance $\sigma_{e}^{2}$, and the definition of $y_{n f}(\boldsymbol{\theta})[n]$ for the square wave actuator signal 72 is given by

$$
y_{n f}(\boldsymbol{\theta})[n]=\frac{4}{\pi} C_{\gamma}\left(f_{i}\right) \sum_{m=1}^{Q\left(T_{s}\right)} \frac{\left|G_{f_{i}, f_{e}}\left(i[2 m-1] \omega_{c}, \boldsymbol{\theta}\right)\right|}{2 m-1} \sin \left([2 m+1] \omega_{c} n T_{s}+\alpha_{m}\right),
$$

in which $\alpha_{m}=\angle\left|G_{f_{i}, f_{e}}\left(i[2 m-1] \omega_{c}, \boldsymbol{\theta}\right)\right|$. Based on $(73)$, an asymptotically efficient estimate for $\boldsymbol{\theta}_{0}$ is defined through [28]

$$
\hat{\boldsymbol{\theta}}_{N}=\arg \min _{N} \frac{1}{N} \sum_{n=1}^{N}\left(y_{D}[n]-y_{n f}(\boldsymbol{\theta})[n]\right)^{2},
$$

where $y_{n f}(\boldsymbol{\theta})$ is defined in (74). In Appendix A the expressions for $u_{D}$ and $y_{n f}(\boldsymbol{\theta})[n]$ are given for a sinusoidal actuator signal. Replacing the expressions (72), 73 with the ones in Appendix A defines the estimate 75 for the sinusoidal case.

We remark that the scaling introduced in the previous section should also be applied to the measured data set $Z_{N}$. For a detailed explanation on how identification can be used for scaled systems, we refer the reader to 34, pages 6-7.

As shown in [28, $\hat{\boldsymbol{\theta}}_{N}$ is asymptotically (i.e. for large $N$ ) normally distributed with mean $\boldsymbol{\theta}_{0}$ and a covariance matrix $\boldsymbol{P}_{\boldsymbol{\theta}}$ whose inverse is given by the Fisher 
Information Matrix:

$$
\boldsymbol{P}_{\boldsymbol{\theta}}^{-1}=\frac{N}{\sigma_{e}^{2}} \bar{E}\left[\nabla_{\boldsymbol{\theta}} y_{n f}(\boldsymbol{\theta}) \nabla_{\boldsymbol{\theta}}^{T} y_{n f}(\boldsymbol{\theta})\right]_{\boldsymbol{\theta}=\boldsymbol{\theta}_{0}},
$$

where $\bar{E}(\cdot)=\frac{1}{N} \sum_{n=1}^{N} E(\cdot)$ is a modified expectation operator and $\nabla_{\boldsymbol{\theta}}$ the gradient operator of $\boldsymbol{\theta}$. This matrix can be rewritten using Parseval's theorem as

$\boldsymbol{P}_{\boldsymbol{\theta}}^{-1}\left[\Phi_{u_{D}}\right]=\frac{N T_{s}}{2 \pi \sigma_{e}^{2}} \int_{-\pi / T_{s}}^{\pi / T_{s}}\left[\nabla_{\boldsymbol{\theta}} G_{f_{i}, f_{e}}(i \omega, \boldsymbol{\theta})\right]_{\boldsymbol{\theta}=\boldsymbol{\theta}_{0}}\left[\nabla_{\boldsymbol{\theta}} G_{f_{i}, f_{e}}(i \omega, \boldsymbol{\theta})\right]^{H}{ }_{\boldsymbol{\theta}=\boldsymbol{\theta}_{0}} \Phi_{u_{D}}(\omega) d \omega$,

and shows that the covariance matrix is a functional in $\Phi_{u_{D}}$, the power spectrum of $u_{D}[n]$. Superscript $H$ denotes the Hermitian conjugate. Note that in 77 the discrete-time transfer function $G_{d}$ is replaced by its continuous-time counterpart $G_{f_{i}, f_{e}}(i \omega)$ since their frequency response are identical in the frequency range up to the Nyquist rate $\pi / T_{s}$.

The covariance matrix depends on the ratios $f_{i}$ and $f_{e}$, the input signal $u_{D}$ via its power spectrum, and on the number of data samples $N$. Using this expression, we will be able to design the input spectrum that provides us the maximal information about the unknown parameter. This optimal spectrum can e.g. be defined as the spectrum that delivers parameter estimates with predetermined upper bounds on the parameter variances using the shortest experiment length (i.e. using the smallest $N$ possible). This input spectrum selection is called optimal experiment design and will be the subject of the next sections. Note also the importance of the gradient of the transfer function, $\nabla_{\boldsymbol{\theta}} G_{f_{i}, f_{e}}(i \omega)$, in (77). This components of this gradient are depicted in Figs. 7 and 8 .

Note that, from (77), we see that in order to calculate the covariance matrix, we need to know the unknown vector $\boldsymbol{\theta}_{0}$, which we aim to estimate! In practice, this vector is replaced by an initial guess that may or may not be derived from a prior experiment. Although this will change the optimal input signal, it has been shown in e.g. 34 that accurate estimates can nonetheless be obtained. 


\section{Direct Method}

In the Direct Method we will also apply a square wave or sinusoidal excitation signal $r(t)$ to the system via the actuator. However, we will not use this signal as input for the identification, but we will use the measured inlet pressure $p_{i}(t)$ instead. The output signal remains to be the outlet pressure and is measured in the same way as in the Indirect Method, yielding $y_{D}[n](n=1, \ldots, N)$. This method is also followed in [1. The input signal is transformed into a discrete-time signal $u_{D}[n]$ using the same measurement mechanism (anti-aliasing filter and sampling with $T_{s}$ time steps). Since the signal $p_{i}(t)$ is measured (and not directly applied as $r(t)$ ), we are not able to retrieve precisely the inlet pressure (the measurements are indeed corrupted by noise). The signal entering the system is thus not known exactly, and consequently, we are facing an errors-in-variables (EIV) identification problem that may lead to serious problems in practice [35, 36]. As shown in [37] on another problem, a way to deal with this EIV problem is to apply a two-stage strategy [38]. First, identify a model $\hat{L}$ of the relation $L_{f_{i}, f_{e}}$ (c.f. 67) between the actuator signal and the noise-corrupted measurements of $p_{i}(t)$. Second, the actuator signal $r(t)$ is filtered by that model $\hat{L}$ to lead to new input data. These new input data converge asymptotically to the noise-free inlet pressure if a good model structure is chosen for the identification. While this method alleviates the EIV issue, it will nevertheless increase the variance of the estimate $\hat{\boldsymbol{\theta}}_{N}$. For the sake of comparison between the Direct and Indirect Method, we will disregard here this increase of variance and suppose that we can obtain the noise-free discretetime inlet pressure using this procedure. This is the same as supposing that we know precisely the filter $L(s)=L_{f_{i}, f_{e}}\left(s, \boldsymbol{\theta}_{0}\right)$.

For a square wave signal $r(t)$, the discrete-time signal $p_{i}[n]$ reads

$u_{D}[n]=\frac{4}{\pi} C_{\gamma}\left(f_{i}\right) \sum_{m=1}^{Q\left(T_{s}\right)}\left|L_{f_{i}, f_{e}}\left(i[2 m-1] \omega_{c}, \boldsymbol{\theta}_{0}\right)\right| \frac{\sin \left([2 m-1] \omega_{c} n T_{s}+\angle L_{f_{i}, f_{e}}\left(i[2 m-1] \omega_{c}, \boldsymbol{\theta}_{0}\right)\right)}{2 m-1}$.

The output signal can therefore also be written as 73 with $y_{n f}(\boldsymbol{\theta})[n]$ now 
defined as

$y_{n f}(\boldsymbol{\theta})[n]=\frac{4}{\pi} C_{\gamma}\left(f_{i}\right) \sum_{m=1}^{Q\left(T_{s}\right)}\left|L_{f_{i}, f_{e}}\left(i[2 m-1] \omega_{c}, \boldsymbol{\theta}_{0}\right)\right| \frac{\left|G_{f_{e}}\left(i[2 m-1] \omega_{c}, \boldsymbol{\theta}\right)\right|}{2 m-1} \sin \left([2 m+1] \omega_{c} n T_{s}+\alpha_{m}\right)$,

where $\alpha_{m}=\angle G_{f_{e}}\left(i[2 m-1] \omega_{c}, \boldsymbol{\theta}\right)+\angle L_{f_{i}, f_{e}}\left(i[2 m-1] \omega_{c}, \boldsymbol{\theta}_{0}\right)$. Based on the above expressions, we can use the data $Z_{N}=\left\{u_{D}[n], y_{D}[n]\right\}_{n=1, \ldots, N}$ to estimate $\hat{\boldsymbol{\theta}}_{N}$ using (75) but now with the new definitions of $y_{n f}(\boldsymbol{\theta})[n]$. Note that we now identify the parameter vector in the transfer function $G_{f_{e}}$ while the considered transfer function was $G_{f_{i}, f_{e}}$ for the Indirect Method. The covariance matrix for the estimate using the Direct Method is also given by $(77)$ but with the same replacement and using the power spectrum corresponding to (78).

We remark that for the Direct Method we have assumed zero noise on the inlet pressure signal. This is never the case in practice and we therefore require a two-stage method. The variance in the parameters will consequently be larger than given by (77). These are strong arguments to prefer the Indirect Method which is the new approach we propose in this paper.

We furthermore point out that the zero noise assumption on the inlet pressure signal is alleviated in the literature 1 by averaging points of the signal $p_{i}(t)$ over a three-sample window to cancel out the measurement noise. This downsampling of data leads to information loss since high frequency dynamics is discarded, which leads to higher parameter variances.

\section{Experiment Design using the Direct Method}

In the previous section we have defined the identification experiment and the identification criterion. Suppose now that our objective is to obtain estimates of both parameters with a variance that is smaller than a given threshold, i.e.,

$$
\begin{aligned}
\operatorname{var}\left(\hat{k}_{N}\right) & =\boldsymbol{e}_{1}^{T} \boldsymbol{P}_{\boldsymbol{\theta}} \boldsymbol{e}_{1} \leq c_{k}, \\
\operatorname{var}\left(\hat{\phi}_{N}\right) & =\boldsymbol{e}_{2}^{T} \boldsymbol{P}_{\boldsymbol{\theta}} \boldsymbol{e}_{2} \leq c_{\phi},
\end{aligned}
$$

where the covariance matrix is the inverse of 777$)$. We would like to optimise the frequency content and the amplitude of the excitation signal $r(t)$ as well as 
In this section we address this problem for the Direct Method. For the sake of brevity, we only derive the solution for the square wave actuator signal (22). The case of a sinusoidal excitation signal (1) can be treated in a similar fashion. Furthermore, we recall that, even though the excitation signal $r(t)$ is induced by the actuator, the signals that are used for the identification are the reconstructed inlet pressure $p_{i}(t)$ and the measured outlet pressure $p_{e}(t)$. The transfer function that is to be identified is thus $G_{f_{e}}\left(s, \boldsymbol{\theta}_{0}\right)$.

The spectrum of the sampled inlet pressure signal with amplitude $C_{\gamma}\left(f_{i}\right)$ and cycle frequency $\omega_{c}$ is given by

$\Phi_{u_{D}}(\omega)=\frac{16}{\pi^{2}} \frac{\pi C_{\gamma}^{2}\left(f_{i}\right)}{2 T_{s}} \sum_{m=1}^{Q\left(T_{s}\right)} \frac{\left|L_{f_{i}, f_{e}}\left(i[2 m-1] \omega_{c}, \boldsymbol{\theta}_{0}\right)\right|^{2}}{(2 m-1)^{2}} \sum_{l=\{-1,1\}} \delta\left(\omega-l[2 m-1] \omega_{c}\right)$.

Since the filter $L_{f_{i}, f_{e}}\left(s, \boldsymbol{\theta}_{0}\right)$ is not identified but only $G_{f_{e}}(s, \boldsymbol{\theta}), L_{f_{i}, f_{e}}$ for the Direct Method is not a function of $\boldsymbol{\theta}$ anymore, but assumed known and therefore always evaluated at $\boldsymbol{\theta}=\boldsymbol{\theta}_{0}$. Substitution of 82 in the expression of the covariance matrix (77) and replacing $G_{f_{i}, f_{e}}$ with $G_{f_{e}} 62$ result in the inverse of the covariance matrix for the Direct Method:

$$
\begin{array}{r}
\boldsymbol{P}_{\boldsymbol{\theta}}^{-1}\left[\omega_{c}, C_{\gamma}\left(f_{i}\right), f_{i}, f_{e}\right]=\frac{16}{\pi^{2}} \frac{N C_{\gamma}^{2}\left(f_{i}\right)}{2 \sigma_{e}^{2}} \sum_{m=1}^{Q\left(T_{s}\right)} \frac{\left|L_{f_{i}, f_{e}}\left(i[2 m-1] \omega_{c}, \boldsymbol{\theta}_{0}\right)\right|^{2}}{(2 m-1)^{2}} \times \\
\operatorname{Re}\left\{\left[\nabla_{\boldsymbol{\theta}} G_{f_{e}}\left(i[2 m-1] \omega_{c}, \boldsymbol{\theta}\right)\right]_{\boldsymbol{\theta}=\boldsymbol{\theta}_{0}} \cdot[\text { C.C. }]\right\},
\end{array}
$$

where C.C. stands for complex conjugate.

\subsection{Optimization Problem and Its Solution}

Due to the amplitude limitation of the actuator, the optimal experiment design problem for the Direct Method is defined mathematically as

$$
\min _{\omega_{c}, C_{\gamma}\left(f_{i}\right), f_{i}, f_{e}} \text { Experiment length } N
$$


subject to

$$
\begin{aligned}
\operatorname{var}\left(\hat{k}_{N}\right) & =\boldsymbol{e}_{1}^{T} \boldsymbol{P}_{\boldsymbol{\theta}}\left[\omega_{c}, C_{\gamma}\left(f_{i}\right), f_{i}, f_{e}\right] \boldsymbol{e}_{1} \leq c_{k}, \\
\operatorname{var}\left(\hat{\phi}_{N}\right) & =\boldsymbol{e}_{2}^{T} \boldsymbol{P}_{\boldsymbol{\theta}}\left[\omega_{c}, C_{\gamma}\left(f_{i}\right), f_{i}, f_{e}\right] \boldsymbol{e}_{2} \leq c_{\phi}
\end{aligned}
$$

in which $c_{k}$ and $c_{\phi}$ are the scaled user-imposed constraints on respectively the variance of the estimates $\hat{k}_{N}$ and $\hat{\phi}_{N}, \boldsymbol{e}_{i}$ the $i$-th unit vector, and $\boldsymbol{P}_{\boldsymbol{\theta}}$ the inverse of matrix (83). The optimization problem for a sinusoidal actuator signal is defined and solved in Appendix B.

We wish to ensure that the variance of the estimates in $\hat{\boldsymbol{\theta}}_{N}=\left(\hat{k}_{N}, \hat{\phi}_{N}\right)^{T}$ do not exceed their respective bounds $c_{k}$ and $c_{\phi}$ using the smallest experiment length. The solution is found as follows:

1. Set the factor $\gamma=\gamma_{m}$ in the actuator amplitude $C_{\gamma}\left(f_{i}\right)$.

2. Define the functions

$$
\begin{aligned}
& N_{k}\left(f_{i}, f_{e}, \omega_{c}\right)=\frac{1}{c_{k}} \boldsymbol{e}_{1}^{T}\left(\frac{\boldsymbol{P}_{\boldsymbol{\theta}}^{-1}\left[\omega_{c}, C_{\gamma_{m}}\left(f_{i}\right), f_{i}, f_{e}\right]}{N}\right)^{-1} \boldsymbol{e}_{1}, \\
& N_{\phi}\left(f_{i}, f_{e}, \omega_{c}\right)=\frac{1}{c_{\phi}} \boldsymbol{e}_{2}^{T}\left(\frac{\boldsymbol{P}_{\boldsymbol{\theta}}^{-1}\left[\omega_{c}, C_{\gamma_{m}}\left(f_{i}\right), f_{i}, f_{e}\right]}{N}\right)^{-1} \boldsymbol{e}_{2},
\end{aligned}
$$

where $\boldsymbol{P}_{\boldsymbol{\theta}}^{-1}$ is given by 83 and we note that $\gamma$ has been replaced by $\gamma_{m}$.

3. Using the functions from the previous item, define

$$
N_{\min }\left(f_{i}, f_{e}\right)=\min _{\omega_{c}}\left\{\max \left[N_{k}\left(f_{i}, f_{e}, \omega_{c}\right), N_{\phi}\left(f_{i}, f_{e}, \omega_{c}\right)\right]\right\} .
$$

4. The optimal experiment length is given by

$$
N_{\text {opt }}=N_{\text {min }}\left(f_{i, o p t}, f_{e, o p t}\right), \text { where }\left\{f_{i, o p t}, f_{e, o p t}\right\}=\arg \min _{f_{i}, f_{e}} N_{m i n}\left(f_{i}, f_{e}\right) .
$$

The solution to the problem is given by the quartet $N_{o p t}, f_{i, o p t}, f_{e, o p t}$, and $\omega_{c, \min }\left(f_{i, \text { opt }}, f_{e, \text { opt }}\right)=\arg N_{\min }\left(f_{i, \text { opt }}, f_{e, \text { opt }}\right)$.

In step 1 the coefficient $\gamma$ in the actuator amplitude $C_{\gamma}\left(f_{i}\right)$ is set to $\gamma=\gamma_{m}$. Equation 83 shows that the parameter variances scale inversely proportional to $C_{\gamma}\left(f_{i}\right)$. Thus, selecting the largest possible amplitude of the actuator is a 
requirement to find the minimal experiment time. The actuator amplitude is now only a function of $f_{i}$. In step 2 the functions $N_{k}$ and $N_{\phi}$ respectively define the required experiment length to ensure that (i) $\operatorname{var}\left(\hat{k}_{N}\right)=c_{k}$ and (ii) $\operatorname{var}\left(\hat{\phi}_{N}\right)=c_{\phi}$. Smaller values than $N_{k}$ and $N_{\phi}$ violate the respective constraints in (86), whereas larger ones lead to longer experiment lengths than required. Both constraints are honoured simultaneously, i.e. satisfying situation (i) and (ii), with the function $N_{\min }\left(f_{i}, f_{e}\right)$ calculated in step 3 . It returns the minimal required experiment length as a function of $f_{i}$ and $f_{e}$ by finding the optimal cycle frequency. The last step then finds the optimal values of $f_{i}$ and $f_{e}$ such that the global minimum of $N_{\min }\left(f_{i}, f_{e}\right)$, i.e. $N_{o p t}$, is obtained.

Previously it was mentioned that in order to compute the optimal input signal the true parameter vector $\boldsymbol{\theta}_{0}$ should be used. However, it is this parameter that we want to identify. Unfortunately, this so-called chicken-and-egg problem is unavoidable in (optimal) Experiment Design. In order to design an optimal signal, some knowledge of the system (in this case the true parameter vector) is required. Consequently, to solve the optimization problem 84 - 86 , we require a prior estimate or an initial estimate $\boldsymbol{\theta}_{\text {init }}$ to evaluate the inverse of the covariance matrix 83. This substitution inevitably leads to suboptimal experiment lengths. However, a recent study [34] showed that much better estimates can be obtained using Experiment Design compared to an arbitrary selection of signals and frequencies, and that in many cases the variance constraints are still honoured. In [39, 40, 41] advanced techniques to circumvent this issue are discussed.

\subsection{Numerical Results}

In this section we follow the four steps defined in the previous section to numerically find the solution $(90)$ that solves $(84)-(86)$. To this end, we grid the frequency $\omega_{c} \in\left[0, \pi / T_{s}\right]$ and ratios $f_{i} \in[0.05,20]$, and $f_{e} \in[0.05,20]$. The grid resolutions are respectively $\Delta \omega_{c}=0.05, \Delta f_{i}=\Delta f_{e}=0.05$. The minimum (maximum) values in the interval of $f_{i}$ and $f_{e}$ correspond to unscaled 
maximum (minimum) inlet and outlet lengths of respectively $L_{i}=L_{e}=0.2$ meters $\left(L_{i}=L_{e}=0.5 \mathrm{~mm}\right)$. Values outside these intervals are assumed to be practically infeasible. The physical system is defined through the values in Table 1. the experiment conditions and experiment design values in Table 2. We wish to ensure that the standard deviation of the estimate $\hat{k}_{N}$ and $\hat{\phi}_{N}$ are respectively less than or equal to $5 \%$ of their true values $k_{0}$ and $\phi_{0}$, defined in Table 1 , using minimal experiment time. We remark that the optimal experiment lengths found in this section depend strongly on the choice of noise variance $\sigma_{e}^{2}$ and actuator amplitude bound $\gamma_{m}$. Thus, comparison with results in the existing literature is only fair under the same experiment conditions.

We first discuss separate parameter estimation results, followed by joint parameter estimation results.

\subsubsection{Separate Parameter Estimation}

Separate parameter estimation refers to the situation where either $k_{0}$ or $\phi_{0}$ is known, and respectively $\phi_{0}$ and $k_{0}$ is unknown and needs to be estimated. We thus wish to ensure that the standard deviation of the estimate $\hat{k}_{N}$ or $\hat{\phi}_{N}$ is less than or equal to $5 \%$ of their true value $k_{0}$ or $\phi_{0}$ in minimal time. Separate estimation is a special case of the situation considered in the previous section where both parameters are identified together (joint estimation), and the optimal experiment design problem in this case can thus be solved following a very similar procedure of the one presented in the previous section. This procedure is given in Appendix B for the case of the sinusoidal excitation signal. The problem and solution for a square wave excitation signal can also be trivially formulated using that appendix.

The different results for separate estimation of $k$ and $\phi$ with sinusoidal or square wave excitation are summarized in Table 3 . In order to interpret these results, it makes sense to start discussing the result corresponding to the sinusoidal excitation and in particular the function $N_{\min }\left(f_{i}, f_{e}\right)$ defined for this signal in B.4 in Appendix B. This function is represented in Fig. B.13 of Appendix B for the case where the parameter $k$ is identified. We observe the 
following:

- For any value of $f_{i}$ the required experiment length reduces with increasing $f_{e}$. Inspection of $N_{\min }\left(f_{i}, f_{e}\right)$ (c.f. (B.4) ) shows that increasing $\partial G_{f_{e}}\left(i \omega_{e x}, \theta\right) / \partial \theta$ decreases $N_{\text {min }}$. From Fig. 5 we see that increasing $f_{e}$ results in a larger absolute value of the derivative. At $f_{e}=20$ we find a maximum at $\omega_{e x} \approx 2$.4. Similar behaviour is shown for the case $\theta=\phi$. The optimal value is $f_{e, o p t}=20$.

- For any value of $f_{e}$ the required experiment length reduces with decreasing $f_{i}$. Let us analyse this result. Note first that $f_{i}$ is only present in the term $C_{\gamma_{m}}\left(f_{i}\right)\left|L_{f_{i}, f_{e}}\left(i \omega, \boldsymbol{\theta}_{0}\right)\right|$, i.e. the amplitude of the inlet pressure $p_{i}(t)$. Obviously, by decreasing $f_{i}$, we increase the inlet volume and thus also the maximal amplitude of the actuator $C_{\gamma_{m}}\left(f_{i}\right)$ (see remark below (3) and (71)), which in turn decreases the required experiment time (c.f. (B.4)). However, the inlet amplitude attenuation due to the filter $\left|L_{f_{i}, f_{e}}\right|$ decreases with decreasing $f_{i}$, yet increases with frequency. It is easy to show that

$$
\forall f_{i}, f_{e}: \max _{\omega} C_{\gamma_{m}}\left(f_{i}\right)\left|L_{f_{i}, f_{e}}\left(i \omega, \boldsymbol{\theta}_{0}\right)\right|=\gamma_{m} \phi_{s} .
$$

Thus, the maximal amplitude of the inlet pressure is equal to $\gamma_{m} \phi_{s}$ and independent of $f_{i}$ and $f_{e}$. However, for small $f_{i}$ this asymptotic value is reached quicker at lower frequencies than for larger $f_{i}$, see Fig. 10. It is also reached quicker for larger $f_{e}$. This explains why one should opt for taking $f_{i, o p t}=0.1$ for any $f_{e}$. It is also at low frequencies where the derivative of $G_{f_{e}}(i \omega, \boldsymbol{\theta})$ with respect to $k$ is large, as explained in the previous item.

- The optimal ratios are $f_{i, o p t}=0.1$ and $f_{e, o p t}=20$. The corresponding optimal experiment length $N_{o p t}=2.86 \times 10^{5}$ at optimal frequency $\omega_{o p t}=$ 2.45 , see Table 3 . The value of $f_{e, \text { opt }}$ corresponds with a choice made in literature [1]: minimising the outlet volume. The optimal values occur at the boundary of the considered intervals of $f_{i}$ and $f_{e}$. The aim in practice is thus to maximise the inlet volume and minimise the outlet volume. 


\begin{tabular}{l|l|l}
\hline Quantity & Scaled Value & Real Value \\
\hline Sampling time & $\tilde{T}_{s}=0.021$ & $T_{s}=0.01 \mathrm{~s}$ \\
Sensor noise variance & $\tilde{\sigma}_{e}^{2}=0.05$ & $\sigma_{e}^{2}=1.25 \times 10^{9} \mathrm{~Pa}^{2}$ \\
Permeability variance constraint & $\tilde{c}_{k}=2.5 \times 10^{-3}$ & $c_{k}=2.5 \times 10^{-3} k_{0}^{2} \mathrm{~m}^{4}$ \\
Porosity variance constraint & $\tilde{c}_{\phi}=2.5 \times 10^{-3}$ & $c_{\phi}=2.5 \times 10^{-3} \phi_{0}^{2}$ \\
Actuator amplitude constraint & $\gamma_{m}=0.1$ & $\gamma_{m}=0.1$ \\
\hline
\end{tabular}

Table 2: The experiment is designed using the above quantities. The scaled system is defined through the choices $L=0.05, k_{s}=k_{0}, \phi_{s}=\phi_{0}$. These scalars define the scaled system in Section 2.2

- Quantitatively the same results and analysis holds for the case where $\theta=$ $\phi$. The experiment lengths in Table 3 show that porosity is more difficult to estimate than permeability, as $\partial G_{f_{e}} / \partial \phi$ is smaller than $\partial G_{f_{e}} / \partial k$ for all values of $f_{e}$ depicted in Fig. 6 .

- Square wave actuator signals result in shorter experiment lengths than sinusoidal ones, in agreement with our result in Appendix E.

\subsubsection{Joint Parameter Estimation}

We follow the procedure outlined at the start of this section, using the same experiment conditions, to analyse the joint parameter estimation experiment design solutions. The optimization problem is given by (84)-(86). The results are also given in Table 4 for the case of the sinusoidal and square wave signals. The logarithm of the $N_{\min }\left(f_{i}, f_{e}\right)$ (c.f. 89$)$ ) is shown in Fig. 9 as a function of $f_{i}$ and $f_{e}$. Starting once again the discussion with the sinusoidal signal, we observe the following:

- The optimal input signal for joint estimation, defined through 90, is found for $\omega_{\text {opt }}=\omega_{c, \min }\left(f_{i, \text { opt }}, f_{e, \text { opt }}\right)=2.35$ at optimal ratios $f_{i, \text { opt }}=0.1$ and $f_{e, o p t}=1.0$, see Table 3 and Fig. 9. The minimal experiment length for this combination is $N_{o p t}=4.47 \times 10^{7}$, and corresponds to an unscaled experiment time of about five days $\left(T_{s}=0.01\right.$ seconds). The experiment 


\begin{tabular}{l|ll|ll|ll}
\hline \multirow{2}{*}{$\begin{array}{l}\text { Direct } \\
\text { Method }\end{array}$} & \multicolumn{2}{|c}{$k$} & \multicolumn{2}{c}{} & \multicolumn{2}{c}{$k, \phi$} \\
& Sine & SW & Sine & SW & Sine & SW \\
\hline$N_{\text {opt }}$ & $2.86 \times 10^{5}$ & $1.66 \times 10^{5}$ & $3.46 \times 10^{5}$ & $2.0 \times 10^{5}$ & $4.47 \times 10^{7}$ & $2.51 \times 10^{7}$ \\
$\omega_{\text {opt }}$ & 2.45 & 2.3 & 2.45 & 2.3 & 2.35 & 2.1 \\
$f_{i, \text { opt }}$ & 0.1 & 0.1 & 0.1 & 0.1 & 0.1 & 0.1 \\
$f_{e, \text { opt }}$ & 20 & 20 & 20 & 20 & 1.0 & 1.0 \\
\hline
\end{tabular}

Table 3: Optimal experiment lengths, scaled frequencies, and optimal ratios $f_{i, \text { opt }}, f_{e, \text { opt }}$ of input using the Direct Method for sinusoidal (Sine) and square wave (SW) actuator signal. Three cases are shown: estimation of only $k$ or $\phi$, and the joint estimation of the parameters $\{k, \phi\}$.

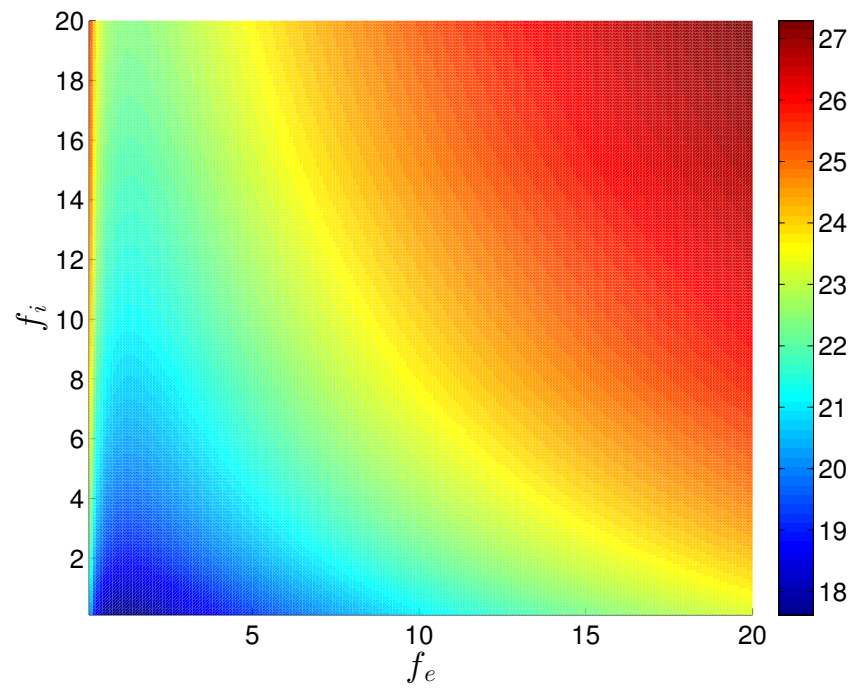

Figure 9: The logarithm minimal experiment length $N_{\min }\left(f_{i}, f_{e}\right)$ 90 for the simultaneous estimation of $k$ and $\phi$ using a sinusoidal excitation signal required to honour their respective variance constraint and the amplitude constraint $\gamma_{m}=0.1$, is plotted against the ratios $f_{i}$ and $f_{e}$. In sharp contrast to single parameter estimation experiments, the optimal ratio $f_{e, o p t}=1.0$. The optimal inlet ratio $f_{i, o p t}=0.1$. 


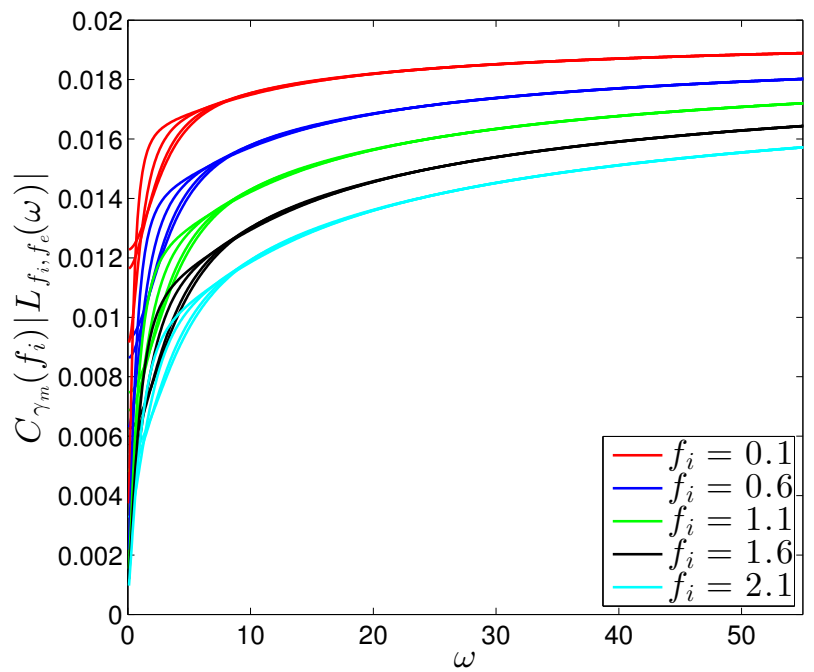

Figure 10: The inlet pressure amplitude $C_{\gamma_{m}}\left(f_{i}\right)\left|L_{f_{i}, f_{e}}\left(i \omega, \boldsymbol{\theta}_{0}\right)\right|$ as a function of frequency $\omega$ for various values of $f_{i}$. For each ratio $f_{i}$ the inlet amplitude is shown for various values of $f_{e}$ in the same color. For the same $f_{i}$ value, smaller values of $f_{e}$ correspond to smaller amplitudes. Observe that a smaller $f_{i}$ leads to a higher amplitude at any given frequency, although all ratios of $f_{i}$ lead to the same asymptotic value of $\gamma_{m} \phi_{s}=0.02$. Notice that $f_{e}$ only affects the amplitude at low frequencies and after $\omega=7.0$ all ratios $f_{e}$ yield the same amplitude at their respective value of $f_{i}$. 
length when using a square wave actuator signal is shorter than when using a sinusoidal actuator signal, as expected from the result in Appendix D.

- For any value of $f_{e}$ it is clear from the figure that by lowering $f_{i}$ shorter experiment times are obtained. This effect has already been explained in Section 4.2.1.

- In sharp contrast to the single parameter experiments (see Table 3), we observe that now, instead, $f_{e}$ should be chosen equal to $f_{e}=f_{e, \text { opt }}=1.0$ as opposed to $f_{e} \gg 1$. The experiment length $N$ is affected by $f_{e}$ through the gradient of $G_{f_{e}}$, see 83 . In Figs. 5 and 6 we see that both components of this gradient increase with increasing $f_{e}$, which in principle should shorten $N$. This is not the result we obtain from the optimal experiment design results.

In order to understand the curiosity mentioned in the last item, i.e. why the optimal ratio is now $f_{e, \text { opt }}=1.0$ instead of $f_{e}=20$, we need to analyse the physical system further. In fact, we will now show that the parameters $k$ and $\phi$ are not identifiable in the limit $f_{e} \rightarrow \infty$. To this end, we introduce the general condition for identifiability $([42,28])$

$$
G\left(i \omega, \boldsymbol{\theta}_{1}\right)=G\left(i \omega, \boldsymbol{\theta}_{2}\right) \forall \omega \Rightarrow \boldsymbol{\theta}_{1}=\boldsymbol{\theta}_{2}
$$

Taking the limit $f_{e} \rightarrow \infty$ of the frequency response $G_{f_{e}}(i \omega, \boldsymbol{\theta})$ in 62 yields

$$
\lim _{f_{e} \rightarrow \infty} G_{f_{e}}(i \omega, \boldsymbol{\theta})=\frac{1}{\cosh \sqrt{i \omega \phi / k}}
$$

Suppose that $\boldsymbol{\theta}_{1}=\left[k_{1}, \phi_{1}\right]^{T}$ and $\boldsymbol{\theta}_{2}=\left[\kappa k_{1}, \kappa \phi_{1}\right]^{T}$, where $\kappa \in \mathbb{R}$. Substitution in 92 then shows that $G_{\infty}\left(i \omega, \boldsymbol{\theta}_{1}\right)=G_{\infty}\left(i \omega, \boldsymbol{\theta}_{2}\right)$ for any frequency $\omega$, whereas $\boldsymbol{\theta}_{1} \neq \boldsymbol{\theta}_{2}$.

We have thus shown that in the limit of $f_{e} \rightarrow \infty$ the transfer function $G_{f_{e}}$ contains the quotient $\frac{\phi}{k}$. As a consequence, infinitely many equal quotients exist by multiplying $k$ and $\phi$ with the same scalar $\kappa$. This problem does not exist for the estimation of only one parameter, as the other one is known. How is this effect then reflected in $(90)$ ? 
It turns out that the components of the gradient $\nabla_{\boldsymbol{\theta}} G_{f_{e}}(i \omega, \boldsymbol{\theta})$ are linearly dependent for all frequencies in the limit $f_{e} \rightarrow \infty$. Consequently, the inverse of the covariance matrix $\boldsymbol{P}_{\boldsymbol{\theta}}^{-1}$ is rank deficient for any input spectrum $\Phi_{u_{D}}(\omega)$. To see this, consider the derivative of the transfer function 62 with respect to the parameters $k$ and $\phi$; see 63) and (64). Dividing the numerator and denominator of each derivative by $f_{e}^{2}$ and taking the limit $f_{e} \rightarrow \infty$ shows that

$$
\frac{\partial G_{f_{e}}(i \omega, \theta)}{\partial k}=-\frac{k}{\phi} \frac{\partial G_{f_{e}}(i \omega, \theta)}{\partial \phi} .
$$

This shows that the elements in $\nabla_{\boldsymbol{\theta}} G_{f_{e}}(i \omega, \boldsymbol{\theta})$ are linearly dependent at all frequencies. Consequently, for any input spectrum $\Phi_{u_{D}}$, the inverse of the covariance matrix for the Direct Method in this limit, i.e.

$\boldsymbol{P}_{\boldsymbol{\theta}}^{-1}=\frac{N T_{s}}{2 \pi \sigma_{e}^{2}}\left(\begin{array}{cc}k^{2} / \phi^{2} & -k / \phi \\ -k / \phi & 1\end{array}\right) \int_{-\pi / T_{s}}^{\pi / T_{s}}\left|\frac{\partial G_{f_{e}}(i \omega, \boldsymbol{\theta})}{\partial \phi}\right|_{\boldsymbol{\theta}=\boldsymbol{\theta}_{0}}^{2}\left|L_{f_{i}, f_{e}}\left(i \omega, \boldsymbol{\theta}_{0}\right)\right|^{2} \Phi_{u_{D}}(\omega) d \omega$

is rank deficient for all $\omega$. Indeed, we find that $\operatorname{det}\left(\boldsymbol{P}_{\boldsymbol{\theta}}^{-1}\right)=0$. It is clear that the variances of the estimates $\hat{k}_{N}$ and $\hat{\phi}_{N}$ approach infinity when increasing $f_{e}$ towards the limit $f_{e} \rightarrow \infty$.

\subsubsection{Discussion}

The above calculations show that when identifying the two parameters jointly, high values of $f_{e}$ should be avoided. We see now that there are two competing mechanisms. One the one hand, the sensitivity of the physical system with respect to the parameters increases with increasing $f_{e}$, which is beneficial to reduce the individual variances of the parameters (and thus shorten the required experiment time). On the other hand, joint estimation of the two parameters becomes more difficult as the derivatives become more and more similar for large $f_{e}$. Hence, the parameters become increasingly correlated for increasing $f_{e}$ for any excitation frequency (or, in fact, any input spectrum), making the covariance matrix singular in the limit $f_{e} \rightarrow \infty$. This effect already plays a dominant role at $f_{e} \approx 20$, as can be observed from Figs. 5 and 6 . Consequently, a trade-off between these two mechanisms has to be made. Optimal experiment 
design shows that the best trade-off is reached by choosing $f_{e}=1.0$ and using an optimal frequency of $\omega_{c, o p t}=2.1$. Due to this trade-off, the minimal experiment time increases to $N=2.51 \times 10^{7}$ (approximately five days), which is two orders of magnitude larger than the experiment lengths obtained for separate parameter estimation.

\section{Experiment Design using the Indirect Method}

In this section we consider experiment design for the Indirect Method. We recall that we estimate the parameters in the transfer function $G_{f_{i}, f_{e}}\left(s, \boldsymbol{\theta}_{0}\right)=$ $L_{f_{i}, f_{e}}\left(s, \boldsymbol{\theta}_{0}\right) G_{f_{e}}\left(s, \boldsymbol{\theta}_{0}\right)$. The input in this case is the actuator signal $r(t)$ and the output is the outlet pressure $p_{e}(t)$, see Fig. 4. We use the same core sample values and scaling as in the previous section, see Tables 1 and 2 . As in Section 4. we only derive the results for the square wave signal. The result for the sinusoidal excitation can be treated in a similar fashion. The experiment design theory on single parameter estimation can be found in Appendix C.

The power spectrum of the square wave actuator signal, corresponding to the input signal 72 , is defined by

$$
\Phi_{u_{D}}(\omega)=\frac{16}{\pi^{2}} \frac{\pi C_{\gamma}^{2}\left(f_{i}\right)}{2 T_{s}} \sum_{m=1}^{Q\left(T_{s}\right)} \frac{1}{(2 m-1)^{2}} \sum_{l=\{-1,1\}} \delta\left(\omega-l[2 m-1] \omega_{c}\right) .
$$

Substitution of this spectrum in the covariance matrix (77) yields

$$
\begin{aligned}
& \boldsymbol{P}_{\boldsymbol{\theta}}^{-1}\left[\omega_{c}, C_{\gamma}\left(f_{i}\right), f_{i}, f_{e}\right]=\frac{16}{\pi^{2}} \frac{N C_{\gamma}^{2}\left(f_{i}\right)}{2 \sigma_{e}^{2}} \times \\
& \sum_{m=1}^{Q\left(T_{s}\right)} \frac{1}{(2 m-1)^{2}} \operatorname{Re}\left\{\left[\nabla_{\boldsymbol{\theta}} G_{f_{i}, f_{e}}\left(i[2 m-1] \omega_{c}, \boldsymbol{\theta}\right)\right]_{\boldsymbol{\theta}=\boldsymbol{\theta}_{0}} \cdot[\text { C.C. }]\right\},
\end{aligned}
$$

where C.C. stands for complex conjugate and the transfer function $G_{f_{i}, f_{e}}$ is given by 68 . As in the previous section, we search for the minimal identification length $N$ that nonetheless fulfils variance constraints on the estimates, and respects the actuator amplitude limitations, by finding optimal values for $\gamma, \omega_{c}$, $f_{i}$, and $f_{e}$. 


\subsection{Optimization Problem and Its Solution}

The optimization problem in the Indirect Method is given by

$$
\min _{\omega_{c}, f_{i}, f_{e}, C_{\gamma}\left(f_{i}\right)} \text { Experiment length } N
$$

subject to

$$
\begin{aligned}
\operatorname{var}\left(\hat{k}_{N}\right) & =\boldsymbol{e}_{1}^{T} \boldsymbol{P}_{\boldsymbol{\theta}}\left[\omega_{c}, C_{\gamma}\left(f_{i}\right), f_{i}, f_{e}\right] \boldsymbol{e}_{1} \leq c_{k}, \\
\operatorname{var}\left(\hat{\phi}_{N}\right) & =\boldsymbol{e}_{2}^{T} \boldsymbol{P}_{\boldsymbol{\theta}}\left[\omega_{c}, C_{\gamma}\left(f_{i}\right), f_{i}, f_{e}\right] \boldsymbol{e}_{2} \leq c_{\phi},
\end{aligned}
$$

in which the inverse of the covariance matrix for two parameters is given by (96) for the transfer function $G_{f_{i}, f_{e}}$ (68). Notice that the optimization problem is similar to 84-86, although we now consider the transfer function is $G_{f_{i}, f_{e}}$ and the amplitude of the input is different.

The solution is found by following the same reasoning as in Section 4.1. We follow the steps 1-4 in Section 4.1, where we instead use 96 for $\boldsymbol{P}_{\boldsymbol{\theta}}^{-1}$.

\subsection{Numerical Results}

Let us now investigate the experiment design solutions, where we use the same physical set-up, experiment parameters, and gridding method as defined in Section 4.2. For convenience we recall that we wish to ensure that the variance of the estimate $\hat{k}_{N}$ and $\hat{\phi}_{N}$ are respectively less than or equal to $5 \%$ of their true values $k_{0}$ and $\phi_{0}$, defined in Table 1. All parameters defining the porous medium are also mentioned in this table. Other parameters are defined in Table 2 We will first consider separate parameter estimation experiment design results, followed by the joint parameter results.

\subsubsection{Separate parameter estimation}

In Appendix C the optimization problem (B.1)- B.2 and solution are given for the separate parameter estimation problem in case of a sinusoidal signal.

The case of a square wave input signal is trivially formulated and solved with 21 the use of 96. 
The optimal experiment times are tabulated in Table 4 The function $N_{\min }\left(f_{i}, f_{e}\right)$ (c.f. (C.2) plotted against $f_{i}$ and $f_{e}$ is qualitatively similar to the Direct Method case (see Fig. B.13 for both $\theta=k$ and $\theta=\phi$. Furthermore, we observe the following:

- For both $\theta=k$ and $\theta=\phi$ the optimal ratios are $f_{i, o p t}=0.1$ and $f_{e, o p t}=$ 20. Apparently, it is best practice to maximize the inlet volume and minimize the outlet volume.

- From C.2 we find that $f_{e}$ influences the gradient $\partial G_{f_{i}, f_{e}}\left(i \omega_{e x}, \theta\right) / \partial \theta$. The larger the gradient, the smaller $N_{\min }\left(f_{i}, f_{e}\right)$ for any value of $f_{i}$. Figures 7 and 8 show that increasing $f_{e}$ increases the gradient of both $k$ and $\phi$. This explains why one should opt for maximising $f_{e}$.

- The same figures also show that decreasing $f_{i}$ decreases the gradient, which increases the required experiment length. Yet, we see from Table 4 that $f_{i}$ should be minimised. The actuator amplitude $C_{\gamma_{m}}\left(f_{i}\right)$ scales inversely proportional to $f_{i}$, and so minimising $f_{i}$ increases the amplitude and reduces the required experiment length. The two effects are thus competing, but the latter one is dominant. Hence, $f_{i}$ should me minimised.

- The optimal excitation frequencies lie close to those values where the gradients are maximal, see Figs. 7 and 8 .

\subsubsection{Joint parameter estimation}

The solution for the joint estimation of permeability and porosity is also presented in Table 4 and is discussed now. The numerical results are obtained through the same procedure as in Section 4 , but using (96) to define $\boldsymbol{P}_{\boldsymbol{\theta}}$. We discuss here directly the case of the square wave signal:

- The function $N_{\min }\left(f_{i}, f_{e}\right)$ attains its minimum for $f_{i, o p t}=0.6$ and $f_{e, o p t}=$ 6.1 , giving $N_{o p t}=N_{\min }(0.6,6.1)=1.8 \times 10^{6}(5$ hours $)$ and an optimal cycle frequency of $\omega_{c, o p t}=1.0$, see Fig. 11 . 


\begin{tabular}{|c|c|c|c|c|c|c|}
\hline \multirow{3}{*}{$\begin{array}{l}\text { Indirect } \\
\text { Method }\end{array}$} & \multirow{2}{*}{\multicolumn{2}{|c|}{$k$}} & \multirow{2}{*}{\multicolumn{2}{|c|}{$\phi$}} & \multirow{2}{*}{\multicolumn{2}{|c|}{$k, \phi$}} \\
\hline & & & & & & \\
\hline & Sine & SW & Sine & SW & Sine & SW \\
\hline$N_{\text {opt }}$ & $3.00 \times 10^{5}$ & $1.74 \times 10^{5}$ & $3.6 \times 10^{5}$ & $2.1 \times 10^{5}$ & $3.3 \times 10^{6}$ & $1.8 \times 10^{6}$ \\
\hline$\omega_{\text {opt }}$ & 2.55 & 2.4 & 2.5 & 2.4 & 1.2 & 1.0 \\
\hline$f_{i, o p t}$ & 0.1 & 0.1 & 0.1 & 0.1 & 0.6 & 0.6 \\
\hline$f_{e, o p t}$ & 20 & 20 & 20 & 20 & 6.4 & 6.1 \\
\hline
\end{tabular}

Table 4: Scaled optimal experiment lengths, frequencies, and ratios $f_{i}, f_{e}$ of input for the Indirect Method. The scaled system is defined through Table 1 These scalars define the scaled system in Section 2.2 For simultaneous estimation in case of a SW actuator signal, note that $f_{i, o p t}$ and $f_{e, o p t}$ translate into an inlet length of $L_{i}=L / 3 \approx 1.7 \mathrm{~cm}$ and an outlet length of $L_{e}=L / 30 \approx 1.7 \mathrm{~mm}$, both of which are feasible in practice.

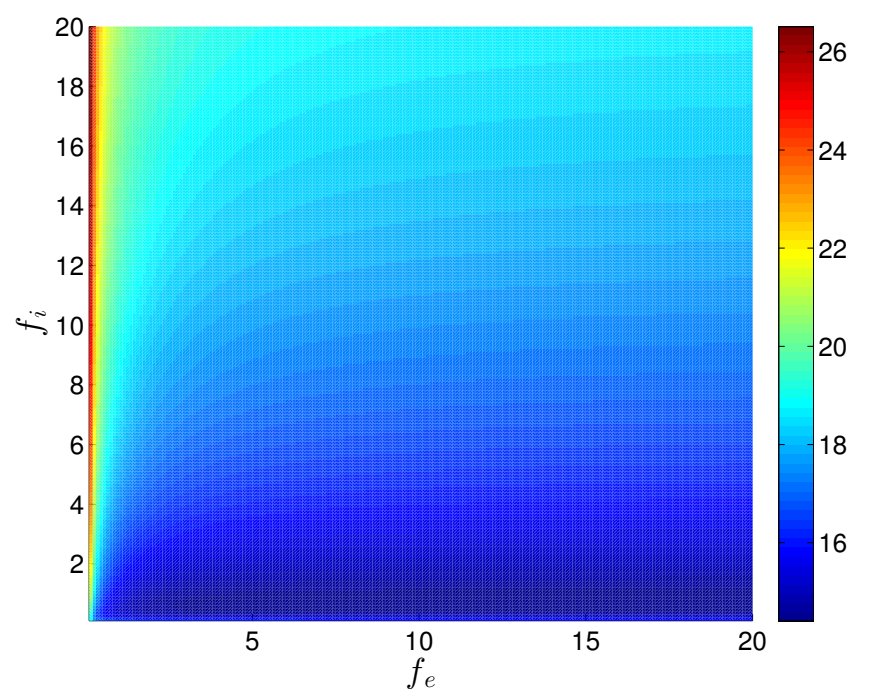

Figure 11: The logarithm of the minimal experiment length $N_{\min }\left(f_{i}, f_{e}\right)$ as a function of the ratios $f_{i}$ and $f_{e}$. Note that the shortest experiment length is obtained for $f_{i, o p t}=0.6$, $f_{e, o p t}=6.1$. 
- These optimal values differ significantly from the separate estimation results, see Table 4 . From Figs. 7 and 8 we see that for $f_{i}=0.1, f_{e}=20$ the gradients of $k$ and $\phi$ are very similar, resulting in high correlation between the two parameters in a joint estimation. This increases the required experiment length. However, we have here no identifiability problem as for the Direct Method.

- In Section 5.2.1 it is explained that the actuator amplitude is maximised by minimising $f_{i}$ and therefore for those cases $f_{i, o p t}=0.1$. By virtue of the previous item, a trade-off between parameter correlation and amplitude maximisation has to be made. We see from Figs. 7 and 8 that the gradients are large and not identical at the optimal ratios of $f_{i}$ and $f_{e}$, so the increase in $f_{i}$ (reducing the amplitude and thus increasing the required experiment length) is compensated by larger gradients.

\subsubsection{Discussion}

We now discuss some differences between the Indirect and Direct Method results.

Let us first discuss the case of separate estimation. For this specific case, observe from Tables 3 and 4 that permeability or porosity is estimated with slightly shorter experiment times when using the Direct Method. However, we point out that we have assumed the input signal $p_{i}(t)$ is noise free, corresponding to the assumption that we know the filter $L_{f_{i}, f_{e}}\left(i \omega, \boldsymbol{\theta}_{0}\right)$. In reality, this is not the case, and will increase the variance of the estimates. Probably the Indirect Method is then preferred.

Let us now go to the more interesting case of joint estimation of the two parameters. Experiment Design has revealed that the optimal experiment length strongly depends on the selected input signals. Indeed, the experiment time for the Indirect Method is a factor fourteen smaller for joint estimation compared to the Direct Method. We have seen for the latter method that $f_{e}$ had to be reduced due to singularity issues at $f_{e}>>1$. This back-off reduces both the inlet pressure amplitude and the gradients, hereby increasing the required 
experiment length enormously compared to the separate estimations. For the Indirect Method, the combination $\left(f_{i}=0.1, f_{e}=20\right)$ corresponds to highly correlated parameters and thus a long experiment time. However, we found that for $f_{i, o p t}=0.6$ and $f_{e, o p t}=6.1$ the gradients can be increased significantly even though the actuator amplitude is less. The difference in the required experiment length for joint estimation compared to the separate estimations is therefore less drastic than in the Direct Method.

\section{Simulation of the Experiment Set-Up: Estimation Results}

In this last section we apply the optimal square wave input signal of the Indirect Method detailed in Section 5. Table 4, to the core sample. All parameters are defined in Tables 1 and 2. In the absence of a physical set-up, we instead generate numerical experiment data $Z_{N}=\left\{u_{D}[n], y_{D}[n]\right\}_{n=1, \ldots, N}$ by applying our optimal input signal $u_{D}$ (using the values in Table 4 and $(72)$ ) and generating noise-corrupted output sequences $y_{D}$ using (74) and (73). We then follow the estimation procedure for the Indirect Method detailed in Section 3

We consider the simultaneous estimation of permeability and porosity from a single experiment. We performed five thousand Monte Carlo simulations yielding the estimated pairs $\left\{\hat{k}_{N}, \hat{\phi}_{N}\right\}$. The optimal square wave signal has a cycle frequency of $\omega_{c, o p t}=1.0$, the experiment length is $N_{o p t}=1.8 \times 10^{6}$, and the optimal ratio $f_{i, o p t}=0.6, f_{e, o p t}=6.1$, see Section 5. A scatter plot of the estimates is shown in Fig. 12 The mean of all points is $(1.0,1.0)$, corresponding to the true scaled parameter values $k_{0}=\phi_{0}=1.0$. The variance of the estimates $\hat{k}_{N}$ and $\hat{\phi}_{N}$ are respectively $\sigma_{k}^{2}=2.49 \times 10^{-3}$ and $\sigma_{\phi}^{2}=2.5 \times 10^{-3}$. The variance constraints defined in Table 2 are clearly respected.

In conclusion, we see the optimal input signal and set-up conditions indeed generate estimates that respect the variance constraints that we set prior to the experiment.

We note that in the experiment design sections we have made use of the fact 
that we know $\boldsymbol{\theta}_{0}$ in order to find the optimal input spectra, since the inverse of the covariance matrix depends on $\boldsymbol{\theta}_{0}$. Unfortunately, this dependence is universal in experiment design: no optimal experiment can be designed without prior knowledge of the system. This work considers a non-Bayesian Experiment Design approach; the unknown true parameter vector $\boldsymbol{\theta}_{0}$ in the inverse covariance matrix should therefore be replaced by an initial estimate $\boldsymbol{\theta}_{\text {init }}$. We have shown in 34] that by replacing the true vector by an initial guess nonetheless delivers better results than an arbitrary input signal or degrees-of-freedom in the set-up. Other solutions to tackle this problem are reported in [39, 41]. It is worthwhile to remark that this problem has a close analog in the Bayesian Experiment Design approaches. Indeed, in these approaches a prior has to be defined for each parameter through e.g. assuming a uniform distribution [13]. Another method, known as "preposterior data analysis" [26], uses a collection of simulated data sets that account for all possible experiment outcomes. This set is used to find the best experiment set-up given that you do not know the actual data prior to the experiment.

We furthermore note that our results are based on the values $k_{0}$ and $\phi_{0}$ shown in Table 1. Different values will lead to different optimal frequencies and optimal ratios $f_{i, o p t}$ and $f_{e, o p t}$. However, the Indirect Method will remain a better estimation method than the Direct Method, regardless of the actual values of permeability and porosity.

\section{Discussion and Conclusions}

In this paper we have introduced a novel estimation method that allows finding the minimal experiment time that is required to estimate permeability and porosity under user-specified parameter variance constraints and actuator limits. We illustrated our methodology on the Dynamic Darcy Cell. We approached the problem by introducing a classical least-squares estimation procedure, from which we derived an expression for the covariance matrix of the estimates. This expression allowed computation of the optimal input signal (either a sinusoid 


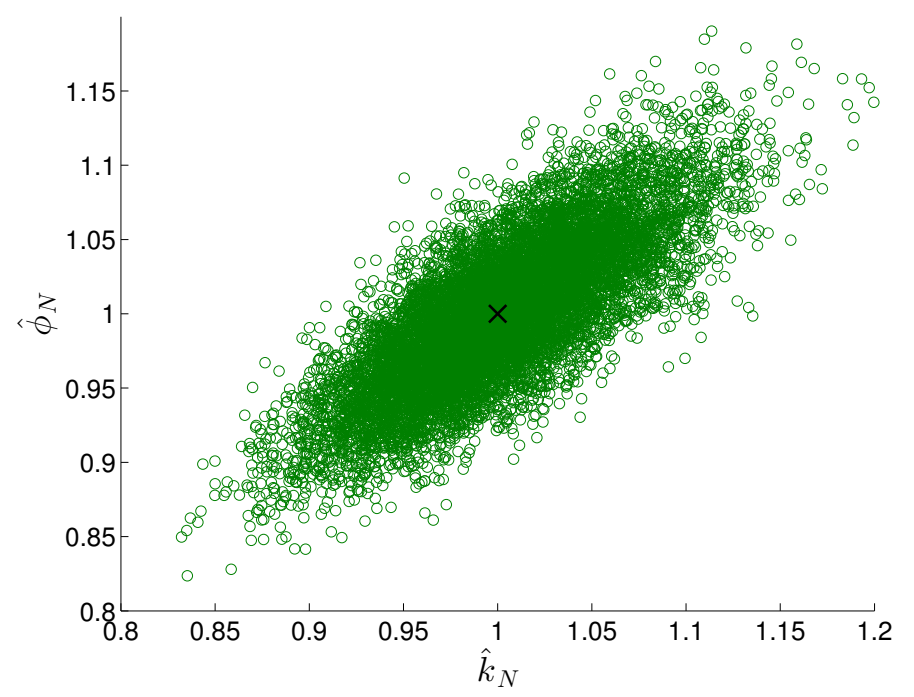

Figure 12: Scatter plot of the couples $\left\{\hat{k}_{N}, \hat{\phi}_{N}\right\}$ resulting from $5 \times 10^{3}$ experiments. The variance of permeability is $\sigma_{k}^{2}=9.9183 \times 10^{-5}$ and of porosity $\sigma_{\phi}^{2}=5.1211 \times 10^{-5}$. The black cross indicates the coordinate of the mean values of permeability and porosity, which were $\left\langle\hat{k}_{N}\right\rangle=1.0$ and $\left\langle\hat{\phi}_{N}\right\rangle=1.0$ and hence respectively equal to $k_{0}=1.0$ and $\phi_{0}=1.0$.

or square wave) and optimal experiment set-up degrees of freedom, being the ratios of the pore volume to inlet volume, $f_{i}$, and pore volume to outlet volume, $f_{e}$ using Experiment Design techniques. We considered sinusoidal and square wave actuator signals and two measurement types: actuator position/outlet pressure measurements (the Indirect Method, novel in this article) and inlet pressure/outlet pressure measurements (Direct Method).

We have proven that square wave signals deliver shorter experiment lengths than sinusoidal ones under the same variance constraints and actuator limits.

The experiment design results for the Direct Method were as follows. For the joint estimation of permeability and porosity, we found that the optimal ratios $f_{e, o p t}=1.0$ and $f_{i, o p t}=0.1$, in contrast to separate parameter experiments for which $f_{e, o p t}=20$. This curiosity originates from a trade-off between variance reduction of permeability and porosity (which is obtained for high $f_{e}$ values, and 
reduces the variance of each separate parameter) and their correlation (which increases with increasing $f_{e}$, and drives the inverse of the covariance matrix to singularity). As a result, the minimum experiment time in this case is two orders of magnitude larger than for the separate parameter experiments.

We also investigated the Indirect Method, novel in this article. We found it superior to the Direct Method for joint parameter estimation: experiment lengths of a factor fourteen less are found. This difference originates mainly from the fact that, compared to the Direct Method, the sensitivity of the system with respect to changes in permeability and porosity for the Indirect Method is large over a broader range of $f_{i}$ and $f_{e}$ values.

Our analytical results are verified by simulating the Dynamic Darcy cell numerically, and we found excellent agreement between the numerical results and theoretical predictions.

Experiment design, and the introduction of the novel estimation method, clearly have enormous potential in practice. This theoretical work is therefore also an invitation to experimentalists to apply our technique to real cores in a laboratory.

Future work could consist of applying our methodology to a system at the catchment scale, e.g. an aquifer. On this scale, multiple input and output signals are typically available, which can be used to identify the spatially-dependent permeability and porosity values of an inhomogeneous reservoir. This is for instance the case in the Hydrogeological Experiment Site of Poitiers, France, where pumping at several wells of the aquifer is possible ([43, 44]).

For applicability of our methodology to catchment-scale problems several hurdles are to be overcome. First, the experiment design method has to be generalised to multiple-input, multiple-output systems (e.g. an aquifer). Second, our methodology is only suitable for linear models. Consequently, linearisation techniques have to be applied to the governing nonlinear equations of the catchment-scale problem, and possibly a dimensional analysis has to be 
performed to validate whether or not the linear dynamics are sufficient to accurately model the process. A third hurdle is the parameter identifiability issue which, on the catchment scale, is likely much larger than on the core scale. User choices such as the spatial resolution of the problem (determining the number of porosity and permeability values that have to be estimated), and the number of input and output locations will play an important role to address this issue. An interesting option that could aid identifiability and input design is the possibility to gather additional information about the aquifer or reservoir with a method known as Fiber-Optic Distributed Acoustic Sensing ([45, 46]).

\section{References}

[1] K. Heller, H. Bruining, D. Smeulders, Permeability obtained from pressure oscillation experiments, part i, one phase flow, Computational Methods in Water Resources 47 (2002) 201-207.

[2] M. Cardiff, T. Bakhos, P. Kitanidis, W. Barrash, Aquifer heterogeneity characterisation with oscillatory pumping: Sensitivity analysis and imaging potential, Water Resources Research 49 (2013) 5395-5410.

[3] I. Song, J. Renner, Analysis of oscillatory fluid flow through rock samples, International Journal of Geophysics 170 (2007) 195-204.

[4] Y. Wang, R. Knabe, Permeability characterisation on tight gas samples using pore pressure oscillation method, Petrophysics 52 (2011) 437-433.

[5] G. Fischer, The determination of permeability and storage capacity: Pore pressure oscillation method, International Geophysics Series 51 (1992) 187211.

[6] G. Boitnott, Use of complex pore transients to measure permeability of rocks, in: SPE Annual Technical Conference and Exhibition, 1997. 
[7] M. Bernabé, B. Evans, A note on the oscillating flow method for measuring rock permeability, International Journal of Rock Mechanics and Mining Sciences 43 (2006) 311-316.

[8] P. Suri, M. Azeemuddin, M. Zaman, A. Kukreti, J.-C. Roegiers, Stressdependent permeability measurements using the oscillating pulse technique, Journal of Petroleum Science and Engineering 17 (1997) 247-264.

[9] A. Hasanov, M. Batzle, Pore pressure pulsing effects on reservoir transport properties, in: Second International Workshop on Rock Physics, 2013.

[10] R. Kranz, J. Saltzman, J. Blacic, Hydraulic diffusivity measurements on laboratory rock samples using an oscillating pore pressure method, International Journal of Rock Mechanics and Mining Sciences \& Geomechanics Abstracts 27 (1990) 345-352.

[11] V. Gupta, S. Sorooshian, The relationship between data and the precision of parameter estimates of hydrologic models, Journal of Hydrology 81 (1985) $57-77$.

[12] F. Kleissen, M. Beck, H. Wheather, The identifiability of conceptual hydrochemical models, Water Resources Research 26 (1990) 2979-2992.

[13] K. Beven, A. Binley, The future of distributed models: Model calibration and uncertainty prediction, Hydrological Processes 6 (2012) 279-298.

[14] B. Wagner, Siimultaneous parameter estimation and contaminant source characterisation for coupled groundwater flow and contaminant transport modelling, Journal of Hydrology 135 (1992) 275-303.

[15] P. Yapo, H. Gupta, S. Sorooshian, Automatic calibration of conceptual rainfall-runoff models: sensitivity to calibration data, Journal of Hydrology 181 (1996) 23-48.

[16] J. Kool, J. Parker, M. Van Genuchten, Parameter estimation for unsaturated flow and transport models - a review, Journal of Hydrology 91 (1987) $255-293$. 
[17] S. Sorooshian, V. Gupta, J. Fulton, Evaluation of maximum likelihood parameter estimation techniques for conceptual rainfall-runoff models: Influence of calibration data variability and length on model credibility, Water Resources Research 19 (1983) 251-259.

[18] S. Sorooshian, V. Gupta, Automatic calibration of conceptual rainfallrunoff models: The question of parameter observability and uniqueness, Water Resources Research 19 (1983) 260-268.

[19] S. Sorooshian, V. Gupta, The analysis of structural identifiability: Theory and application to conceptual rainfall-runoff models, Water Resources Research 21 (1985) 487-495.

[20] G. Kuczera, Improved parameter inference in catchment models 1. evaluating parameter uncertainty, Water Resources Research 19 (1983) 1151-1162.

[21] G. Kuczera, Improved parameter inference in catchment models 2. combining different kinds of hydrological data and testing their compatability, Water Resources Research 19 (1983) 1163-1172.

[22] P. Mahar, B. Datta, Optimal identification of ground-water pollution sources and parameter estimation, Journal of Water Resources Planning and Management 127 (2001) 20-29.

[23] N.-S. Hsu, W.-G. Yeh, Optimum experimental design for parameter identification in groundwater hydrology, Water Resources Research 25 (1989) 1025-1040.

[24] T. Nishikawa, W.-G. Yeh, Optimum pumping test design for the parameter identification of groundwater systems, Water Resources Research 25 (1989) $1737-1747$.

[25] J. McCarthy, W.-G. Yeh, Optimal pumping test design for parameter estimation and prediction in groundwater hydrology, Water Resources Research 26 (1990) 779-791. 
[26] P. Leube, A. Geiges, W. Nowak, Bayesian assessment of the expected data impact on prediction confidence in optimal sampling design, Water Resources Research 48.

[27] X. Bombois, G. Scorletti, P. Van den Hof, R. Hilderbrand, Least-costly identification experiment for control, Automatica 42 (2006) 1651-1662.

[28] L. Ljung, System Identification: Theory for the User, Prentice Hall, 1999.

[29] R. Aster, B. Borchers, C. Thurber, Parameter Estimation and Inverse Problems, Academic Press, 2005.

[30] J. Bear, Dynamics of Fluids in Porous Media, Dover Publications, 1972.

[31] P. Forchheimer, Wasserbewegung durch boden, Zeit. Ver. Deutsch. Ing. 45 (1901) 1781-1788.

[32] L. Dake, Developments in Petroleum Science 8: Fundamentals of Reservoir Engineering, Elsevier, 1978.

[33] U. S. Lab, Rosetta soil database.

URL http://www . ars .usda.gov/News/docs .htm?docid=8953

[34] M. Potters, X. Bombois, M. Mansoori, P. Van den Hof, Estimating parameters with pre-specified accuracies in distributed parameter systems, Accepted in the International Journal of Control, DOI: 10.1080/00207179.2016.1138143.

[35] T. Söderström, Errors-in-variables methods in system identification, Automatica 43 (939-958).

[36] T. Söderström, L. Wang, R. Pintelon, J. Schoukens, Can errors-in-variables systems be identified from closed-loop experiments, Automatica 49 (2013) 681-684.

[37] M. Mansoori, P. Van den Hof, J. Jansen, D. Rashtchian, Pressure transient analysis of bottomhole pressure and rate measurements using system identification techniques, SPE Journal 20 (2015) 1005-1027. 
[38] P. Van den Hof, R. Schrama, Indirect method for transfer function estimation from closed loop data, Automatica 29 (6) (1993) 1523-1527.

[39] L. Gerencsèr, H. Hjalmarsson, J. Mårtensson, Identification of ARX systems with non-stationary inputs - asymptotic analysis with applications to adaptive input design, Automatica 45 (2009) 623-633.

[40] C. Larsson, M. Annergren, H. Hjalmarsson, C. Rojas, X. Bombois, A. Mesbah, P. Modén, Model predictive control with integrated experiment design for OE systems, in: Proceedings of the 2013 European Control Conference, 2013.

[41] M. Forgione, X. Bombois, P. Van den Hof, Data-driven model improvement for model-based control, Automatica 52 (2015) 118-124.

[42] H. Pohjanpalo, System identifiability based on the power series expansion of the solution, Mathematical Biosciences 41 (1978) 21-33.

[43] A. Chamroo, R. Ouvrard, T. Poinot, G. Porel, B. Nauleau, J. Bodin, Continuous-time model identification of wells interaction on the hydrogeological experimental site of poitiers, in: European Control Conference, 2014.

[44] A. Chamroo, R. Ouvrard, T. Poinot, J. Bodin, B. Nauleau, G. Porel, Hydrogeological experimental site of poitiers (france), in: $17^{t} h$ IFAC Symposium on System Identification, 2015.

[45] J. van der Horst, et. al., Fiber optic sensing for improved wellbore production surveillance, in: International Petroleum Technology Conference, 2014 .

[46] J. Koelman, J. Lopez, J. Potters, Optical fibers: The neurons for future intelligent wells, in: SPE Intelligent Energy International, 2012. 


\section{Appendix A. Definitions using Sinusoidal Excitation Signals}

In this appendix we define the sinusoidal actuator signal $r[n]$ for the Direct and Indirect Method that can be used to estimate the parameters permeability and porosity in Section 3

Sinusoidal Input in the Indirect Method

The power spectrum of a sinusoidal actuator signal, i.e. the input, with amplitude $C_{\gamma}\left(f_{i}\right)$ and frequency $\omega_{e x}$ is defined by

$$
\Phi_{u_{D}}(\omega)=\frac{\pi C_{\gamma}^{2}\left(f_{i}\right)}{2 T_{s}} \sum_{l=\{-1,1\}} \delta\left(\omega-l \omega_{e x}\right) .
$$

This leads to the discrete-time actuator signal $r[n]$, which is the input $u_{D}[n]$, given by

$$
u_{D}[n]=C_{\gamma}\left(f_{i}\right) \sin \left(\omega_{e x} n T_{s}\right)
$$

In this case the noise-free outlet pressure is given by

$$
y_{n f}(\boldsymbol{\theta})[n]=C_{\gamma}\left(f_{i}\right)\left|G_{f_{i}, f_{e}}\left(i \omega_{e x}, \boldsymbol{\theta}\right)\right| \sin \left(\omega_{e x} n T_{s}+\alpha\right),
$$

where $\alpha=\angle\left|G_{f_{i}, f_{e}}\left(i \omega_{e x}, \boldsymbol{\theta}\right)\right|$. Replacing 72 and 73 by respectively A.2 and A.3 defines the estimation problem (75) for the Indirect Method using a sinusoidal actuator signal.

Sinusoidal Input in the Direct Method

The power spectrum the discrete-time inlet pressure signal, defined as the input signal in the Direct Method, as a consequence of a sinusoidal actuator signal with amplitude $C_{\gamma}\left(f_{i}\right)$ and frequency $\omega_{e x}$ is defined by

$$
\Phi_{u_{D}}(\omega)=\frac{\pi C_{\gamma}^{2}\left(f_{i}\right)}{2 T_{s}}\left|L_{f_{i}, f_{e}}\left(\omega_{e x}, \boldsymbol{\theta}_{0}\right)\right| \sum_{l=\{-1,1\}} \delta\left(\omega-l \omega_{e x}\right) .
$$

The discrete-time pressure signal $p_{i}[n]$ is thus given by $u_{D}[n]$ and reads

$$
u_{D}[n]=C_{\gamma}\left(f_{i}\right)\left|L_{f_{i}, f_{e}}\left(i \omega_{e x}, \boldsymbol{\theta}_{0}\right)\right| \sin \left(\omega_{e x} n T_{s}+\angle L_{f_{i}, f_{e}}\left(i \omega_{e x}, \boldsymbol{\theta}_{0}\right)\right),
$$


where the filter $L_{f_{i}, f_{e}}\left(i \omega_{e x}, \boldsymbol{\theta}_{0}\right)$ is defined in (67). The noise-free outlet pressure is then given by

$y_{n f}(\boldsymbol{\theta})[n]=C_{\gamma}\left(f_{i}\right)\left|L_{f_{i}, f_{e}}\left(i \omega_{e x}, \boldsymbol{\theta}_{0}\right)\right|\left|G_{f_{e}}\left(i \omega_{e x}, \boldsymbol{\theta}\right)\right| \sin \left(\omega_{e x} n T_{s}+\angle L_{f_{i}, f_{e}}\left(i \omega_{e x}, \boldsymbol{\theta}_{0}\right)+\angle G_{f_{e}}\left(i \omega_{e x}, \boldsymbol{\theta}\right)\right)$.

Replacing 72 and 73 by respectively A.5 and A.6 defines the estimation problem 75 for the Direct Method.

\section{Appendix B. Single Parameter Experiment Design for Direct Method}

In this appendix we consider the estimation of either permeability of porosity using a sinusoidal actuator signal. The power spectrum of the sinusoidal inlet pressure signal (c.f. A.5) is given by A.4. The true parameter vector is denoted by $\theta_{0}$ and is thus either equal to $k_{0}$ or $\phi_{0}$. To find the minimal experiment time that honours the variance constraint on one of the estimates given the amplitude constraints on the inlet pressure signal reads:

$$
\min _{\omega_{e x}, C_{\gamma}\left(f_{i}\right), f_{i}, f_{e}} \text { Experiment length } N
$$

subject to

$$
\operatorname{var}\left(\hat{\theta}_{N}\right)=P_{\theta}\left[\omega_{e x}, C_{\gamma}\left(f_{i}\right), f_{i}, f_{e}\right] \leq c_{\theta},
$$

where $c_{\theta}$ is either $c_{k}$ (constraint value for permeability) or $c_{\phi}$ (constraint value for porosity), $G_{f_{e}}$ is given by (62), and the inverse of the covariance matrix (??) is given by the scalar

$$
P_{\theta}^{-1}=\frac{N C_{\gamma}^{2}\left(f_{i}\right)}{2 \sigma_{e}^{2}}\left|L_{f_{i}, f_{e}}\left(i \omega_{e x}, \boldsymbol{\theta}\right)\right|^{2}\left|\frac{\partial G_{f_{e}}\left(i \omega_{e x}, \theta\right)}{\partial \theta}\right|_{\theta=\theta_{0}}^{2} .
$$

From this expression it is clear that a requirement to find the optimal solution is that the condition $\operatorname{var}\left(\hat{\theta}_{N}\right) \equiv P_{\theta}=c_{\theta}$ is met, and that furthermore that we should select $\gamma=\gamma_{m}$, see previous sections for motivation. The solution to B.1)-B.2 for given ratios of $\left(f_{i}, f_{e}\right)$ is then trivially found to be

$$
N_{\min }\left(f_{i}, f_{e}\right)=\frac{2 \sigma_{e}^{2}}{c_{\theta} C_{\gamma_{m}}^{2}\left(f_{i}\right)} \min _{\omega_{e x}}\left[\left|L_{f_{i}, f_{e}}\left(i \omega_{e x}, \boldsymbol{\theta}_{0}\right)\right|^{-2}\left|\frac{\partial G_{f_{e}}\left(i \omega_{e x}, \theta\right)}{\partial \theta}\right|_{\theta=\theta_{0}}^{-2}(\mathbf{B} .4)\right.
$$




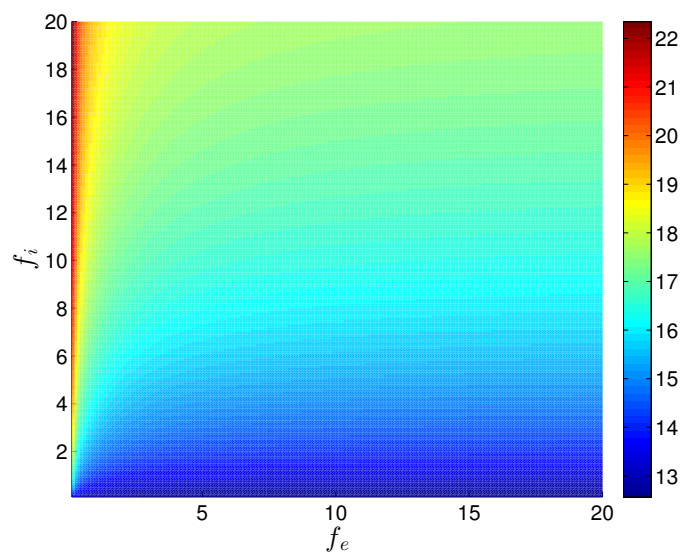

Figure B.13: The logarithm of $N_{\min }\left(f_{i}, f_{e}\right)$ as a function of $f_{i}$ and $f_{e}$ for $\theta=k$. The optimal ratios are $f_{i, o p t}=0.1$ and $f_{e, o p t}=20$.

where the optimal excitation frequency $\omega_{e x, \min }\left(f_{i}, f_{e}\right)$ is a function of $f_{i}$ and $f_{e}$ and is the frequency at which the minimum in (B.4) is obtained. The shortest possible experiment length is then found to be:

$$
N_{\text {opt }}=N_{\text {min }}\left(f_{i, o p t}, f_{e, o p t}\right), \quad\left(f_{i, o p t}, f_{e, o p t}\right)=\arg \min _{f_{i}, f_{e}} N_{\min }\left(f_{i}, f_{e}\right) .
$$

The logarithm of the function $N_{\min }\left(f_{i}, f_{e}\right)$ (c.f. (B.4) $)$ is plotted for $\theta=k$ in Figure B.13 as a function of $f_{i}$ and $f_{e}$. The result for porosity is qualitatively the same. All optimal values are reported in Table 3. All other parameters are defined in Tables 1 and 2 . The constraint value is respectively $c_{\theta}=c_{k}$ and $c_{\theta}=c_{\phi}$ for these cases, see Table 2

\section{Appendix C. Single Parameter Experiment Design for Indirect Method}

In this appendix we follow the exact same procedure as in Appendix B, but now for actuator to outlet measurements. The actuator limit is given by $\gamma_{m}=0.1$. All parameters are defined in Tables 1 and 2. The optimization problem for the Indirect Method is given by $(\overline{B .1})-(\overline{B .2})$, where $P_{\theta}$ should be replaced with

$$
P_{\theta}^{-1}\left[\omega_{c}, C_{\gamma}\left(f_{i}\right), f_{i}, f_{e}\right]=\frac{N C_{\gamma}^{2}\left(f_{i}\right)}{2 \sigma_{e}^{2}}\left|\frac{\partial G_{f_{i}, f_{e}}\left(i \omega_{e x}, \theta\right)}{\partial \theta}\right|_{\theta=\theta_{0}}^{2} .
$$


Following the same arguments as in the previous appendix we find that the minimal experiment time for a given set of $\left\{f_{i}, f_{e}\right\}$ is given by

$$
N_{\min }\left(f_{i}, f_{e}\right)=\frac{2 \sigma_{e}^{2}}{c_{\theta} C_{\gamma_{m}}^{2}\left(f_{i}\right)} \min _{\omega_{e x}}\left|\frac{\partial G_{f_{i}, f_{e}}\left(i \omega_{e x}\right)}{\partial \theta}\right|_{\theta=\theta_{0}}^{-2},
$$

where $G_{f_{i}, f_{e}}$ is defined in (68). The optimal experiment length $N_{\text {opt }}$ is then found as in the previous appendix.

\section{Appendix D. Dynamical Relationship between Inlet Pressure and} Outlet Pressure

An alternative way to find the dynamical relationship between the inlet pressure $p_{i}(t)$ and outlet pressure $p_{e}(t)$ has been considered in [1. In that paper, the expression for the system output when applying an input signal $u(t)=C \cos (\omega t)$ was found to be

$$
y_{n f}(t)=p(x=1, t)=C \frac{\Psi_{1}(\omega) \cos (\omega t)+\Psi_{2}(\omega) \sin (\omega t)}{\Psi_{1}^{2}(\omega)+\Psi_{2}^{2}(\omega)}
$$

$$
\begin{aligned}
& \Psi_{1}(\omega)=\cosh \left(\sqrt{\frac{\omega \phi}{2 k}}\right) \cos \left(\sqrt{\frac{\omega \phi}{2 k}}\right)+\sqrt{\frac{\omega \phi}{2 k}} f \times \\
& {\left[\sinh \left(\sqrt{\frac{\omega \phi}{2 k}}\right) \cos \left(\sqrt{\frac{\omega \phi}{2 k}}\right)-\cosh \left(\sqrt{\frac{\omega \phi}{2 k}}\right) \sin \left(\sqrt{\frac{\omega \phi}{2 k}}\right)\right], } \\
& \Psi_{2}(\omega)=\sinh \left(\sqrt{\frac{\omega \phi}{2 k}}\right) \sin \left(\sqrt{\frac{\omega \phi}{2 k}}\right)+\sqrt{\frac{\omega \phi}{2 k}} f \times \\
& {\left[\sinh \left(\sqrt{\frac{\omega \phi}{2 k}}\right) \cos \left(\sqrt{\frac{\omega \phi}{2 k}}\right)+\cosh \left(\sqrt{\frac{\omega \phi}{2 k}}\right) \sin \left(\sqrt{\frac{\omega \phi}{2 k}}\right)\right] . }
\end{aligned}
$$

It is clear that (58) is more compact and easier to use, especially for more complex input signals. A simple time plot of these two signals shows that the expressions (58) and (D.1) are indeed equivalent for $u(t)=C \cos (\omega t)$ (not shown here). Note that for this comparison, $\sin (\cdot)$ in 58 needs to be replaced by $\cos (\cdot)$. In fact, one can show with trigonometric identities that their amplitude ratio between the outlet and inlet pressure

$$
R=\frac{2}{\sqrt{\Psi_{1}^{2}+\Psi_{2}^{2}}}
$$


is equal to $\left|G_{f_{e}}\right|$ in $[62)$, and that their phase shift $\Theta$ between outlet and inlet pressure

$$
\Theta=\arctan \frac{-\Psi_{2}}{\Psi_{1}}
$$

942 is equal to $\alpha=\angle G_{f_{e}}$.

where $Q\left(T_{s}\right)=\left\lfloor\frac{1}{2}\left(\frac{\pi}{\omega_{c} T_{s}}+1\right)\right\rfloor$.

Lastly, for an input $u(t)$ that is connected to output $y(t)$ through a transfer function $G_{\Xi}\left(i \omega, \boldsymbol{\theta}_{0}\right)$, where $\Xi$ contains all degrees-of-freedom of the set-up, assuming white measurement noise and no feedback, the inverse of the covariance 
matrix of the estimates $\hat{\boldsymbol{\theta}}_{N}$ is given by

$$
\boldsymbol{P}_{\boldsymbol{\theta}}^{-1}\left[\Phi_{u}(\omega)\right]=\frac{N T_{s}}{2 \pi \sigma_{e}^{2}} \int_{-\pi / T_{s}}^{\pi / T_{s}}\left[\nabla_{\boldsymbol{\theta}} G_{\Xi}(i \omega, \boldsymbol{\theta})\right]_{\boldsymbol{\theta}=\boldsymbol{\theta}_{0}}\left[\nabla_{\boldsymbol{\theta}}^{*} G_{\Xi}(i \omega, \boldsymbol{\theta})\right]_{\boldsymbol{\theta}=\boldsymbol{\theta}_{0}} \Phi_{u}(\omega) d \omega,
$$

We are now ready to prove the following lemma.

Lemma 1. Let $N_{1}, \omega_{e x}=\omega_{o p t}, \Xi_{\text {opt }}, C$ be respectively the minimal experiment time, optimal excitation frequency, optimal set of degrees-of-freedom in the set-up, and amplitude $C$ of the sinusoid $u(t)=C \sin \left(\omega_{\text {ex }} t\right)$ that solve the optimization problem

$$
\min _{\omega_{e x}, \Xi} N
$$

subject to

$$
\forall \theta_{i} \in \boldsymbol{\theta}: \operatorname{var}\left(\hat{\theta}_{i, N}\right) \leq c_{\theta_{i}},
$$

where $c_{\theta, i}$ is the variance constraint for estimate $\hat{\theta}_{i, N}$. Then, using a square wave signal with cycle frequency $\omega_{c}=\omega_{\text {opt }}$ and amplitude $C$, the minimal experiment time $N_{S W}$ that fulfil constraint E.5) can be guaranteed to be at least a factor $\pi^{2} / 16$ times smaller than $N_{1}$.

Proof. The expression of the covariance matrix E.3 for a single sinusoid with spectrum (E.1) using the optimal parameters $N=N_{1}, \omega_{e x}=\omega_{o p t}, \Xi=\Xi_{\text {opt }}$, and amplitude $C$ reads

$$
\boldsymbol{P}_{\boldsymbol{\theta}, o p t, 1}^{-1}=\frac{N_{1} C^{2}}{2 \sigma_{e}^{2}} \operatorname{Re}\left\{\left[\nabla_{\boldsymbol{\theta}} G_{\Xi_{o p t}}\left(i \omega_{o p t}, \boldsymbol{\theta}\right)\right]_{\boldsymbol{\theta}=\boldsymbol{\theta}_{0}}\left[\nabla_{\boldsymbol{\theta}} G_{\Xi_{o p t}}\left(i \omega_{o p t}, \boldsymbol{\theta}\right)\right]_{\boldsymbol{\theta}=\boldsymbol{\theta}_{0}}^{*}\right\} .
$$

${ }_{95}$ By definition this covariance matrix honours the variance constraints. The 966 substitution of E.2,$\omega_{c}=\omega_{\text {opt }}$, and $N=N_{S W}$ into the covariance matrix (E.3) results in

$$
\begin{array}{r}
\boldsymbol{P}_{\boldsymbol{\theta}, S W}^{-1}=\frac{16}{\pi^{2}} \frac{N_{S W} C^{2}}{2 \sigma_{e}^{2}} \sum_{m=1}^{Q\left(T_{s}\right)} \frac{1}{(2 m-1)^{2}} \operatorname{Re}\left\{\left[\nabla_{\boldsymbol{\theta}} G_{\Xi_{o p t}}\left(i[2 m-1] \omega_{o p t}, \theta\right)\right]_{\boldsymbol{\theta}=\boldsymbol{\theta}_{0}} \times\right. \\
\left.\left[\nabla_{\boldsymbol{\theta}} G_{\Xi_{o p t}}\left(i[2 m-1] \omega_{o p t}, \boldsymbol{\theta}\right)\right]_{\boldsymbol{\theta}=\boldsymbol{\theta}_{0}}^{*}\right\}
\end{array}
$$




$$
\boldsymbol{P}_{\boldsymbol{\theta}, S W}^{-1}=\frac{16}{\pi^{2}} \frac{N_{S W} C^{2}}{2 \sigma_{e}^{2}} \operatorname{Re}\left\{\left[\nabla_{\boldsymbol{\theta}} G_{\Xi_{o p t}}\left(i \omega_{o p t}, \boldsymbol{\theta}\right)\right]_{\boldsymbol{\theta}=\boldsymbol{\theta}_{0}}\left[\nabla_{\boldsymbol{\theta}} G_{\Xi_{o p t}}\left(i \omega_{o p t}, \boldsymbol{\theta}\right)\right]_{\boldsymbol{\theta}=\boldsymbol{\theta}_{0}}^{*}\right\}
$$
+extra positive definite terms.

Clearly, by choosing $N_{S W}=\frac{\pi^{2}}{16} N_{1}<N_{1}$ and using E.6, E.7, the above equation may be rewritten as

$$
\boldsymbol{P}_{\boldsymbol{\theta}, S W}^{-1}=\boldsymbol{P}_{\boldsymbol{\theta}, o p t, 1}^{-1}+\text { extra positive definite terms. }
$$

What remains to show is that the variance(s) of $\hat{\theta}_{N, i}$ has (have) not increased. To this end, using Schur's complement, we rewrite the constraints in (E.5) for covariance matrix $\boldsymbol{P}_{\boldsymbol{\theta}, S W}^{-1}$ as:

$$
\forall \theta_{i} \in \boldsymbol{\theta}:\left(\begin{array}{cc}
c_{\theta_{i}} & \boldsymbol{e}_{i} \\
\boldsymbol{e}_{i}^{T} & \boldsymbol{P}_{\boldsymbol{\theta}, S W}^{-1}
\end{array}\right) \succeq \mathbf{0}
$$

Denote $\boldsymbol{\Omega} \succeq 0$ the extra positive definite terms in E.8. For the constraint on $\hat{\theta}_{N, i}$, we find by substitution of $\mathrm{E} .8$ that we require

$$
\forall \theta_{i} \in \boldsymbol{\theta}:\left(\begin{array}{cc}
c_{\theta_{i}} & \boldsymbol{e}_{i} \\
\boldsymbol{e}_{i}^{T} & \boldsymbol{P}_{\boldsymbol{\theta}, o p t, 1}^{-1}+\boldsymbol{\Omega}
\end{array}\right)=\left(\begin{array}{cc}
c_{\theta_{i}} & \boldsymbol{e}_{i} \\
\boldsymbol{e}_{i}^{T} & \boldsymbol{P}_{\boldsymbol{\theta}, \text { opt }, 1}^{-1}
\end{array}\right)+\left(\begin{array}{cc}
0 & 0 \\
0 & \boldsymbol{\Omega}
\end{array}\right) \succeq \mathbf{0} .
$$

